\title{
Shape-selective Diffusion of Olefins in 8-Ring Solid Acid
}

\section{Microporous Zeolites}

\author{
An Ghysels ${ }^{a b c}$, Samuel L.C. Moors ${ }^{a b}$, Karen Hemelsoet ${ }^{a}$, Kristof De Wispelaere ${ }^{a}$, \\ Michel Waroquier ${ }^{\mathrm{a}}$, German Sastre ${ }^{\mathrm{cd}}$, and Veronique Van Speybroeck ${ }^{\mathrm{ac}}$ (Dated: \\ September 1, 2015)
}

\begin{abstract}
The diffusion of olefins through 8-ring solid acid microporous zeolites is investigated using molecular dynamics simulations techniques and using a newly developed flexible force field. Within the context of the Methanol to Olefin (MTO) process and the observed product distribution, knowledge on the diffusion paths is essential to obtain molecular level control over the process conditions. Eight-ring zeotype materials are favorably used for the MTO process as they give a selective product distribution towards low carbon olefins. To investigate how composition, acidity and flexibility influence the diffusion paths of ethene and propene, a series of isostructural aluminosilicates (zeolites) and silicoaluminophosphates (AIPOs and SAPOs) are investigated with and without randomly distributed acidic sites. Distinct variations in diffusion of ethene are observed in terms of temperature, composition, acidity, and topology ( $\mathrm{AEI}, \mathrm{CHA}, \mathrm{AFX})$. In general, diffusion of ethene is an activated process for which free energy barriers for individual rings may be determined. We observe ring dependent diffusion behavior which can not solely be described in terms of the composition and topology of the rings. A new descriptor had to be introduced namely the accessible window area (AWA), inspired by implicit solvation models of proteins and small molecules. The AWA may be determined throughout the molecular dynamics trajectories and correlates well with the number of ring crossings at the molecular level and the free energy barriers for ring crossings from one cage to the other. The overall observed diffusivity is determined by molecular characteristics of individual rings for which AWA is a proper descriptor. Temperature-induced changes in framework dynamics and diffusivity may be captured by following the new descriptor throughout the simulations.

\footnotetext{
a Center for Molecular Modeling, Ghent University, Technologiepark 903, 9052 Zwijnaarde, Belgium

b These two authors contributed equally.

c an.ghysels@ugent.be, gsastre@itq.upv.es, veronique.vanspeybroeck@ugent.be

d Instituto de Tecnologia Quimica UPV-CSIC, Universidad Politecnica de Valencia, Av. Los Naranjos s/n, 46022, Valencia, Spain
} 


\section{INTRODUCTION}

Zeolites are among the most widely investigated and topical of inorganic materials, finding extensive use in a wide variety of applications, such as catalysis, separations, ion exchangers, and adsorbents. ${ }^{1}$ These materials have a three-dimensional periodic framework that consists of nanometersized channels and cages providing high porosity and large surface area. At present 218 distinct framework types are described, ${ }^{2}$ of which only a small fraction is widely used as industrial material. ${ }^{3}$ The exploitation of these materials as catalysts has gained a new and green boost with the introduction of methanol and ethanol as base chemicals, produced from alternative feedstocks, which have the additional advantage of being cheaper and potentially renewable. ${ }^{4-8}$ In this area, the methanol-to-olefins (MTO) process is one of the most important alternatives to the oil-based routes. ${ }^{9-11}$ It is now already commercialized at large scale in various places worldwide. ${ }^{10} \mathrm{H}-\mathrm{ZSM}-5$ and SAPO-34 are the archetypal industrially applied catalysts for methanol conversion. H-ZSM-5 has the MFI topology and is an aluminosilicate whereas SAPO-34 has the CHA topology and is a siliocoaluminophosphate material. Both materials give a substantially different product distribution. SAPO-34 is very selective towards ethene and propene, due to its particular topology exhibiting 8-rings, which hinder the diffusion of larger species through the windows. ${ }^{12-14}$ In the search for optimal catalysts for the MTO process, other potential candidates have been explored, which have a tunable ethene/propene ratio and low deactivation rates. ${ }^{10}$ The overall reaction mechanism is nowadays accepted to be based on a hydrocarbon pool (HP), which co-catalyzes the reactions. ${ }^{15-17}$

In this paper we have selected three classes of small ring zeotype materials, bearing the $\mathrm{AEI}, \mathrm{CHA}$ and AFX topology with 8-ring channels at largest, as displayed in Fig. 1. Those topologies have received already some interest within the context of the MTO process and may host bulky aromatic intermediates, which are essential for the conversion of methanol to hydrocarbons. ${ }^{11}$ Indeed it is now well accepted that a hydrocarbon pool mechanism operates in which an organic center is trapped in the zeolite pores and acts as co-catalyst. ${ }^{12,15-19} \mathrm{~A}$ wealth of theoretical studies have appeared to explore potential reaction mechanisms in various catalysts and to understand the material characteristics at the molecular level which control the activity and product selectivity. ${ }^{11,20}$ Various experimental studies have pointed towards the importance of diffusion of product species through the channel systems for 

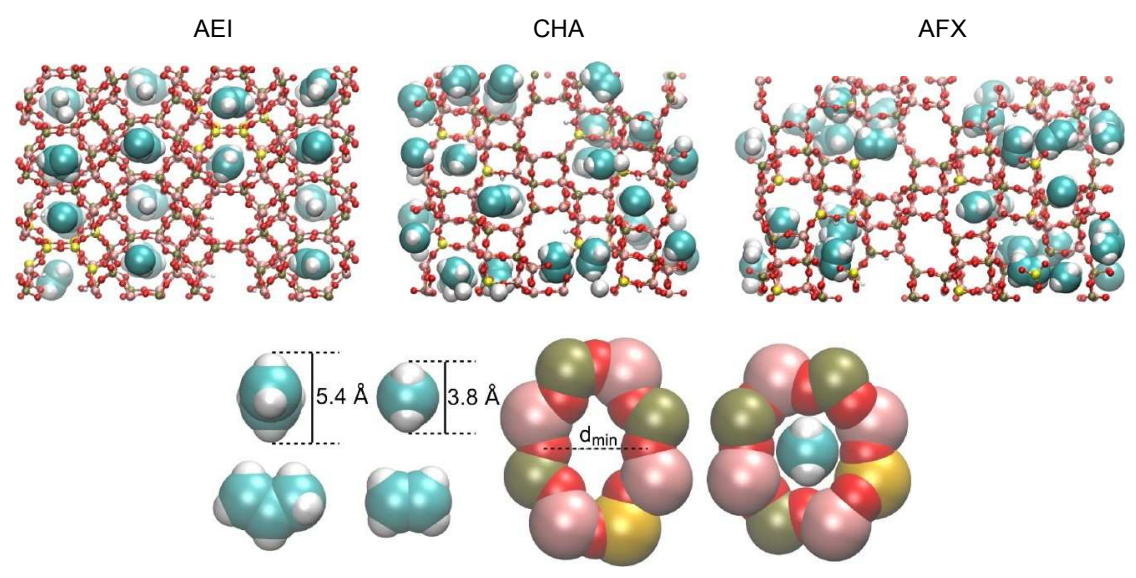

FIG. 1: (Top) Structures of SAP0-18 (AEI), SAP0-34 (CHA), and SAP0-56 (AFX), loaded with ethene molecules. (Bottom left) Van der Waals representations of ethene and propene in front and side orientations. (Bottom middle) An elliptically shaped 8-ring with indicated dmin distance. The ring is too small for ethene to cross through. (Bottom right) An 8-ring with more circular shape that is large enough for ethene to cross through. Atom colors: $C$ (blue), H (white), O (red), Al (pink), Si (yellow), P (taupe).

Theoretical studies of diffusion are usually tackled using simulations based on classical force fields. ${ }^{30}{ }^{32}$ Force field based approaches in combination with MD simulations are now well established. ${ }^{26}$ Using MD, a large portion of the potential energy surface can be sampled. 
Temperature as well as the flexibility of the target molecules and frameworks can be included explicitly. The basic idea of MD is to trace the trajectory of a guest molecule over time. In the long time limit, the slope of the mean square displacement becomes (a multiple of) the diffusion coefficient. This explicit derivation of diffusivities has been extensively studied in literature, e.g. by Awati et al. ${ }^{33}$, Krishna et al. ${ }^{34}$, Smit and Maessen et al. ${ }^{26}$, Garcia and Dubbeldam et al. ${ }^{35}$, O'Malley et al. ${ }^{32}$, Kiel et al. ${ }^{36-39}$ in various materials, for various hydrocarbons. For processes where diffusion barriers are too high, MD runs may be too slow to efficiently generate particle trajectories over the time-scale of the diffusion process. ${ }^{40}$ In such cases other techniques have been suggested to study diffusion, including transitionstate sampling, transition path sampling, ${ }^{41}$ or kinetic Monte Carlo. The interested reader is further referred to the recent work of Abouelnasr and Smit. ${ }^{42}$ In the present paper, we study the diffusion of both ethene and propene in small ring zeolites using MD techniques. Most of the quantitative analysis will be performed on ethene, as for this molecule diffusion is fast enough to obtain meaningful data using conventional MD simulations. In contrast, for propene advanced sampling techniques would be necessary as mentioned above.

The emphasis of the current study particularly lies on the influence of the material's flexibility on the molecular diffusion paths. Indeed, for the studied cases the pore size of the zeolite has the same order of magnitude as the kinetic diameter of the molecules. The influence of framework flexibility on the diffusion properties has long been a matter of debate. $^{26}$ Traditionally, theoretical zeolite diffusion studies have used a rigid zeolite framework to reduce the computational cost or to increase the simulation time needed to obtain sufficient diffusion data. This rigid framework approach does not account for possible guestinduced window deformations. Moreover, unphysical host-guest interactions encountered with rigid frameworks may lead to unrealistic dynamics and aggregate formation of the guest molecules. ${ }^{26,43}$ Recently, a number of MD simulation studies have been published that compare the diffusion in rigid and flexible zeolite frameworks. While in some cases the influence of lattice vibrations was very small, in other studies the introduction of flexiblity led to significantly altered diffusivities. ${ }^{33,35,44}$ Several researchers have cautioned on the accuracy of the flexible model, showing that the diffusion coefficient is highly sensitive to the force field. ${ }^{35,45}$ In comparing the rigid and flexible framework approaches, the influence of the force field can be partly factored out by using a time-averaged zeolite structure in the simulations. Krishna et al. used this approach to study methane diffusion in several 8-ring 
zeolites. ${ }^{34,46}$ They found very similar diffusion coefficients for the simulations with a rigid and flexible zeolite, summarized the link between ring diameter and diffusion coefficient, and confirmed an exponential trend. Awati et al. ${ }^{33}$ investigated the ring diameter distribution in great detail, more specifically ring anharmonicity and ring shape (circular versus elliptical, framed 'dimensionality' in Ref. ${ }^{33}$ ), and identified when diffusion through a flexible framework may be equally well modeled by diffusion through a rigid time-averaged framework. In an effort to maintain the efficiency of the rigid framework approximation while still including some degree of flexibility, they proposed to perform MD on a set of rigid snapshots, which were extracted from simulations of an empty framework. They also studied the diffusion of methane in several 8-ring zeolites, and good agreement with a fully flexible structure was obtained. $^{33}$

For molecules that are smaller than the zeolite windows, framework deformation may cause flattening of the windows from a circular to a more elliptical shape (Fig. 1), thus resulting in decreased diffusion. In the studies of Krishna et al. and Awati et al. described in the previous paragraph, the kinetic diameter of methane is much smaller than the 8-ring dimensions. In such cases an averaged zeolite structure may indeed sufficiently capture ring deformations to represent a fully flexible lattice.

For tight-fitting molecules however, the flexibility of the framework can be expected to facilitate window crossings by temporary widening of the windows. The inverse could also occur, when framework flexibility reduces adsorbate mobility if the widening slows down the dynamics. In this work, the kinetic dimensions of ethene and propene are comparable to or even larger than the 8-ring dimensions (see Fig. 1), which suggests that dynamic expansion of the rings could affect the diffusion process. We have thus opted to model framework flexibility explicitly with a flexible forcefield.

Importantly, the choice for a flexible force field in this work allows us to investigate the ring structural and dynamical properties in detail, for instance the ring diameter, ring eccentricity, and ring puckering amplitude. In this paper, all individual rings are analyzed, and their structure is correlated to the number of crossings through the ring. Indeed, diffusion of small hydrocarbons through the channels of 3-dimensional zeolites may be regarded as a cage hopping process. The central aim is thus to see which factors influence this cage hopping rate: ring composition, ring topology, or a third structural/dynamical factor. Such a detailed analysis has not been performed before, and has the advantage that we can ob- 
serve statistically significant differences between individual rings. In total 54 systems have been simulated using MD techniques. The influence of framework topology, composition and acidity has been accounted for and the flexible forcefield introduced earlier by one of the authors has been adapted to account for all of these factors. The number of ring crossings have been studied at the level of individual rings and in terms of typical characteristics of the ring. A new molecular descriptor is introduced which correlates the accessible window area of the guest species with the number of ring crossings. 


\section{METHODOLOGY}

\section{A. Zeolite frameworks}

Diffusion of ethene and propene is investigated in three topologies using atomistic molecular dynamics. The selected topologies are AEI, CHA, and AFX. These topologies have 8-rings as their largest windows. Two compositions are considered as a starting point: (1) pure $\mathrm{SiO}_{2}$ and (2) pure $\mathrm{AlPO}_{4}$ structures. These pure materials (without acid sites) are listed in Table I: (1) SSZ-39, SSZ-13, and SSZ-16, and (2) AIPO-18, AIPO-34, and AIPO-56. Acid sites are introduced in these structures: (1) some Si are substituted by Al in the pure silica materials (alumino-silicate or zeo materials), and (2) some Al are substituted by Si in the $\mathrm{AIPO}_{4}$ structures (silico-alumino-phosphate or SAPO materials). Additionally, Si-islands are introduced in SAPO materials by substitution of Al-O-P fragments by Si-O-Si. The acid sites and Si-islands are introduced randomly with the program zeoTsites ${ }^{47,48}$ respecting the overall Si/AI/P ratio as described in detail below. The resulting acidic materials (with acid sites) are listed in Table I: (1) H-SSZ-39, H-SSZ-13, and H-SSZ-16 (zeo); (2) SAPO-18, SAPO-34, and SAPO-56 (SAPO).

In the AEI topology, the corresponding zeo and SAPO materials are H-SSZ-39 and SAPO18. For H-SSZ-39 a Si/AI ratio of 5.0 (64 Al, $320 \mathrm{Si}$ and 64 protons in the simulated unit cell) is selected, close to that reported for zeolites employed in the MTO and methanol-tohydrocarbons (MTH) processes. ${ }^{10}$ For SAPO-18, a content around $9.5 \%$ Si (with respect to $\mathrm{Si}+\mathrm{Al}+\mathrm{P})$ is chosen as the experimental benchmark, ${ }^{49}$ with $50 \%$ of the Si substituted through mechanism I $(\mathrm{P} \rightarrow \mathrm{Si}, \mathrm{H})$, and in our case this means 4 Si-islands made of $8 \mathrm{Si}$ atoms each, in a total of $32 \mathrm{Si}, 184 \mathrm{Al}, 168 \mathrm{P}$ and 16 protons in the simulated unit cell.

Our model of CHA as SAPO material (SAPO-34) is taken from Dai et al., ${ }^{23}$ who reported a composition of $1.1 \mathrm{mmol} / \mathrm{g}, 6.0 \mathrm{mmol} / \mathrm{g}$, and $4.1 \mathrm{mmol} / \mathrm{g}$ for $\mathrm{Si}, \mathrm{Al}$ and $\mathrm{P}$ respectively in the final solid. This is similar to work in the group of Olsbye ${ }^{14}$ where an $(A I+P) / S i$ ratio of 11 was reported as optimum for MTO, showing that lower ratios contribute more actively to catalyst deactivation. This ratio corresponds to approximately one acid site (proton) per cage. Katada et al. ${ }^{50}$ reported a ratio of 10 , also along the same values above. For these samples it has been shown that most of the acid sites are isolated and hence no Si-islands are formed. Regarding the alumino-silicate version (H-SSZ-13), the same argument of the 
deactivation is also invoked, with about one proton per cage as the optimum content, and this gives a Si/Al ratio of about $11 .{ }^{14}$ With all this information, our unit cells contain 36 T-sites with the distribution $33 \mathrm{Si}, 3 \mathrm{Al}$ for H-SSZ-13 and $18 \mathrm{AI}, 15 \mathrm{P}, 3 \mathrm{Si}$ for the SAPO-34.

For the AFX systems in zeo (H-SSZ-16) and SAPO (SAPO-56) materials, the following chemical compositions are selected. For H-SSZ-16 a Si/AI ratio of 6 has been reported ${ }^{50}$ for MTO and MTH processes. The Al distribution in the simulated unit cell (56 Al, 328 Si and 56 protons) is created with the requirements of Loewenstein and Dempsey rules commonly applied in zeolites, and these conditions are also imposed to the AEI and CHA systems. For SAPO-56, a content of tetrahedral atoms close to $11.5 \% \mathrm{Si}, 55 \% \mathrm{Al}, 33.5 \% \mathrm{P}$, reported by Wilson et al., ${ }^{51}$ is selected, also taking into account that $64 \%$ of the $\mathrm{Si}$ is isolated and $36 \%$ of the $\mathrm{Si}$ is forming small silicon islands. The simulated SAPO-56 unit cells (188 Al, $48 \mathrm{Si}$, $148 \mathrm{P}$ and 40 protons) contain 3 Si-islands made of $5 \mathrm{Si}$ atoms each and 33 isolated Si.

TABLE I: Overview of selected frameworks and the 54 simulated systems with varying loading (ethene or propene molecules in the supercell) and at different temperatures $\mathrm{T}$.

\begin{tabular}{|c|c|c|c|c|c|}
\hline material & topology & loading & $\mathrm{T}[\mathrm{K}]$ & supercell & \\
\hline SSZ-39 & AEI & 29 eth & $300,450,600$ & $2 \times 1 \times 2$ & \\
\hline SSZ-13 & $\mathrm{CHA}$ & 26 eth & $300,450,600$ & $2 \times 2 \times 2$ & \\
\hline SSZ-16 & AFX & 32 eth & $300,450,600$ & $1 \times 2 \times 2$ & \\
\hline AIPO-18 & AEI & 29 eth & $300,450,600$ & $2 \times 1 \times 2$ & \\
\hline AIPO-34 & $\mathrm{CHA}$ & 26 eth & $300,450,600$ & $2 \times 2 \times 2$ & \\
\hline AIPO-56 & AFX & 32 eth & $300,450,600$ & $1 \times 2 \times 2$ & \\
\hline material & topology & loading & $\mathrm{T}[\mathrm{K}]$ & supercell & $\mathrm{Si} / \mathrm{Al}$ \\
\hline H-SSZ-39 & AEI & 29 eth, 29 prop & $300,450,600$ & $2 \times 1 \times 2$ & 5 \\
\hline H-SSZ-13 & $\mathrm{CHA}$ & 26 eth, 26 prop & $300,450,600$ & $2 \times 2 \times 2$ & 11 \\
\hline \multirow[t]{2}{*}{ H-SSZ-16 } & AFX & 32 eth, 32 prop & $300,450,600$ & $1 \times 2 \times 2$ & 5.58 \\
\hline & & & & & $(\mathrm{Al}+\mathrm{P}) / \mathrm{Si}$ \\
\hline SAPO-18 & AEI & 29 eth, 29 prop & $300,450,600$ & $2 \times 1 \times 2$ & 11 \\
\hline SAPO-34 & $\mathrm{CHA}$ & 26 eth, 26 prop & $300,450,600$ & $2 \times 2 \times 2$ & 11 \\
\hline SAPO-56 & AFX & 32 eth, 32 prop & $300,450,600$ & $1 \times 2 \times 2$ & 7 \\
\hline
\end{tabular}

A supercell consisting of four or six unit cells is used as the periodic simulation box (last 
column of Table I) with volumes of roughly 25000,19000 , and $25000 \AA^{3}$ for AEI, CHA, and AFX respectively. Details about the Si substitution in SAPOs can be found in our previous work, ${ }^{52-54}$ and sample CIF files of the unit cells employed in the calculations are given as Supp. Inf.

\section{B. Adsorbates}

We focus on the diffusion of ethene and propene, which are the main products of the methanol conversion. The onset of methanol conversion is expected not to be limited by diffusion, but at later stages of the methanol conversion, the diffusion of the products may strongly affect the reaction kinetics and shape selectivity of the MTO reaction. ${ }^{23}$ The loadings (see Table I and Fig. 1) are chosen to be about one to two adsorbates per cage. Initial tests with lower loadings of just below one molecule per cage give similar results for the diffusion coefficient as the higher loading simulations (within statistical errors of these shorter trajectories of $20 \mathrm{~ns}$ ). The simulations are therefore performed at high loadings, where more statistics are available because of averaging over the molecules.

\section{Force field}

Most of the earlier MD work on zeolites has been performed using force fields for pure silica zeolites given the large number and high quality of the force fields available. ${ }^{45,55,56}$ Also, the introduction of $\mathrm{Al}$ and protons as well as the specific bridging Si-O-Al oxygens, differing from Si-O-Si oxygens, suggest that shell models might perform better. However, MD have traditionally preferred rigid ion force fields which behave much better in the usual MD algorithms in terms of energy conservation and computer time. ${ }^{26,57}$ In this study, we present a new force field that can handle zeolites as well as alumino-silicates and silicoalumino-phosphates in any $(\mathrm{Si}, \mathrm{Al}, \mathrm{P})$ compositional range, with protons as charge compensating cation, hence specifically designed for Brønsted acid zeotypes. To make the force field compatible with zeolites, AIPOs and SAPOs, accuracy is somewhat sacrificed in favor of generality. Here, we summarize the main traits of this new force field. A more detailed description of the force field development and a list of new parameters are given in Supp. Inf. (Table S3). 
The new force field starts with an approach similar to that employed in a previous work $^{58,59}$ where the nonbonding interactions between the zeolite framework atoms include not only $\mathrm{T}-\mathrm{O}(\mathrm{T}=\mathrm{Si}, \mathrm{Al}, \mathrm{P})$ and $\mathrm{O}-\mathrm{O}$, as in many zeolite force fields, but also T-T terms. Such terms are included with the Lennard-Jones functional rather than the Buckingham functional. The obtained T-T parameters are small, which indicates that in practice we are close to the common values of parameters found in similar force fields. ${ }^{30}$ Also in agreement with previous force fields, the O-O parameters contribute more importantly, and this is the expected result in zeolites where oxygen anions, with a larger size than the $\mathrm{T}$ cations, dominate the interactions. Given the pivotal role of the oxygens, their charges have been differentiated, taken into account their respective chemical local environment, and hence, we have distinguished $\mathrm{O} 1$ for Si-O1(H1)-Al (Brønsted site) and $\mathrm{O} 2$ for Si-O2-Si and Al-O2-P. In the case of $\mathrm{O} 2$, it has been found by calculations in quartz and berlinite that there is no significant accuracy loss by equalizing the oxygens in Si-O-Si and Al-O-P. ${ }^{60}$ Also, employing $\mathrm{O} 2$ in Si-O2-Al where $\mathrm{O} 2$ is not holding a proton (neighbour to Brønsted site) was found a reasonable approximation and, more importantly, introduced a considerable simplicity in a model that would have become complex if too many oxygen types had to be assigned for each system. The corresponding oxygen charges are the only difference between these oxygens as their O-O Lennard-Jones interactions do not distinguish between oxygen types.

Brønsted sites have been considered through a Coulomb subtracted Morse potential, similarly as in a previous work in the groups of Catlow and Sauer. ${ }^{61}$ The current parametrization gives very similar values; however in the current case there was no need to introduce a Buckingham nor Lennard-Jones term between non-bonded O...H atoms as in the previous case. ${ }^{61}$ The $\mathrm{OH}$ term was tuned to give a reasonable $\mathrm{OH}$ distance around $0.96 \AA$, typical for Brønsted sites in zeolites.

Following our previous experience for the three-body terms we have introduced harmonic terms for the following triads: O-Si-O, P-O-Al, Si-O-Al and Si-O-Si, while we have not introduced an O-Al-O term. The values of the constants are similar to those found in other force fields for zeolites. Taking into account that the current force field will be used for dynamics at - among other - high temperatures, improving the harmonic approximation could be a reasonable option, but we have sacrificed accuracy for simplicity, and this is important given the large number of parameters to be determined.

The force field fitting as well as the lattice energy minimisations have been performed 
using GULP. ${ }^{62}$ The forcefield for hydrocarbons (ethene and propene) is taken from Oie et al, ${ }^{63}$ which we have used previously in similar systems with good results. ${ }^{64}$ The structural performance of the new force field in silica polymorphs shows an accuracy of cell parameters within $1 \%$ and cell volumes within $2 \%$ (Supp. Info., Table S5).

\section{Molecular dynamics}

By varying the topology (AEI/CHA/AFX), the composition (with/without acidic sites, zeo/SAPO), the adsorbates (ethene/propene) and the temperature (300/450/600 K), a total of 54 systems are simulated with MD (overview Table I).

The DL_POLY Classic ${ }^{65}$ (version 1.9) simulation package is used for MD simulations in the NVT ensemble. The velocity Verlet integrator is used with a time step of $1 \mathrm{fs}$. Some simulations used a shorter time step of $0.5 \mathrm{fs}$, i.e. for the materials with acid sites at 450 and $600 \mathrm{~K}$. Snapshots of the positions are stored every 1000 time steps. Long-range interactions are calculated with the smoothed particle mesh Ewald method with a cutoff radius between 11.7 and $12.0 \AA$.

Taking into account that many zeolites show negative thermal expansion 66,67 and that this may affect the sizes of the 8-ring windows at the different temperatures simulated in this study (300, 450 and $600 \mathrm{~K}$ ), we have optimized the unit cells (without adsorbates) at different temperatures employing the zero static internal stress approximation as implemented in GULP, ${ }^{68}$ and using a coreshell force field which gives an accurate description of the thermal expansion behavior. ${ }^{69}$ These cell parameters are used in the subsequent NVT MD runs. Volume fluctuations and volume expansion induced by the adsorbates are neglected as the same volume is used independently of the loading. Nevertheless, since the periodic box is a supercell, some unit cell deformation is still possible.

First, an equilibration run of 380000 time steps is performed with the velocity rescaling thermostat. Next, a production run of $210^{8}$ time steps is performed. The MD thus covers $200 \mathrm{~ns}$ for simulations with time step $1 \mathrm{fs}$ and $100 \mathrm{~ns}$ for those with time step $0.5 \mathrm{fs}$. The temperature is controlled with the Nosé-Hoover thermostat with a coupling time constant of 1 ps. This thermostat is known to disturb little the dynamics of the system, which is essential when modeling diffusion. We verified the quality of the production run by tracing the configurational energy and the instantaneous temperature, which both fluctuate around 
the mean, and by tracing the conserved energy quantity, which does not shift significantly during the run.

\section{E. Diffusion coefficients}

The mean square displacement (MSD) of an adsorbate molecule during a time interval T (also called lag time) is a measure for the distance traveled by the molecule. The slope of the MSD as a function of lag time determines the self-diffusion coefficient $D$. From a single MD trajectory, many displacement vectors $\bar{r}_{i}\left(t_{0}+T\right)-\bar{r}_{i}\left(t_{0}\right)$ may be extracted that represent the distance traveled by a molecule $\mathbf{i}$ over the time interval $\mathrm{T}$, starting from an initial time $t_{0}$. The MSD may thus be computed as

$$
\operatorname{MSD}(\mathrm{T})={\frac{1}{N_{\mathrm{ads}}}}_{\mathbf{i}}^{\mathrm{N}_{\mathrm{ads}}} \frac{1}{\mathrm{~N}_{\mathrm{T}}}{ }_{t_{0}}^{\mathrm{N}_{\mathrm{T}}}\left|\overline{\mathrm{r}}_{\mathbf{i}}\left(\mathrm{t}_{0}+\mathrm{T}\right)-\overline{\mathrm{r}}_{\mathbf{i}}\left(\mathrm{t}_{0}\right)\right|^{2}
$$

In Eq. 1, the squared length of this displacement vector is averaged over many $\left(\mathrm{N}_{\mathrm{T}}\right)$ such time intervals, since the initial time $t_{0}$ can freely vary along the trajectory, as long as the total simulation time covers $t_{0}+T$. In addition, averaging over the molecules improves the statistics of the M SD(т) from a single trajectory, and thus also of the self-diffusion constant $\mathrm{D}$, defined according to the so-called Einstein relation,

$$
\operatorname{MSD}(t)=6 D t+b
$$

where $b$ is the offset at time zero. The line is fitted in the lag time range 100 to $3000 \mathrm{ps}$ using a least-sqaure fit. The MD trajectory is split into 20 non-overlapping blocks of equal length. For each of the 20 blocks, an MSD curve is calculated by taking the average over the $\mathrm{N}_{\text {ads }}$ adsorbates, and a diffusion coefficient $\mathrm{D}$ is fit. The reported MSD curves and corresponding D values in Section III are calculated as the average of these 20 MSD curves and $20 \mathrm{D}$ values, respectively, with error bars given by the corresponding standard error. Further computational details are given in Supp. Inf.

F. Ring crossings and free energy profiles

The free energy profile of an ethene molecule passing through an 8-ring is constructed as follows. An ellipse is fit to the atoms of the ring (see Fig. 2). The center of a specific ring is 
determined as the center of this ellipse, while the plane of the ring is the plane of the fitted ellipse. A coordinate is now introduced to determine the position of an ethene molecule with respect to the ring plane. The -axis is defined as the normal to the ellipse plane. The coordinates (center of mass or geometrical center) of an ethene molecule are projected onto the -axis, giving a trajectory $(t)$ in time, which represents the distance of the ethene molecule to the ring plane. When $(\mathrm{t})$ changes sign, a crossing through the ring has taken place. Fig. 2 shows a molecule crossing from one side of the framework ring $(<0)$ to the other side $(>0)$, with $=0$ when it crosses the plane of the ring.

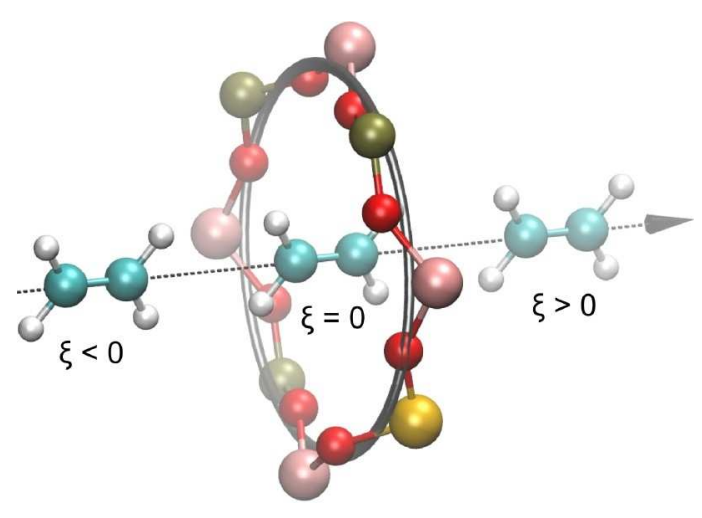

FIG. 2: Definition of -axis, describing the position of the adsorbate molecule with respect to the ring plane; changes sign when the molecule crossing the ring. Three example positions of ethene are drawn: $\quad<0,=0$ (ethene at center of ring), and $>0$.

The probability of crossing relates to the free energy barrier. The normalized histogram of (t) is the probability distribution $\mathrm{P}()$ of the ethene molecule with respect to the axis. For a given ring, is computed at every snapshot for any adsorbate molecule in the neighborhood of this ring. A histogram is computed from all the values by binning the -axis. By taking the logarithm of $\mathrm{P}()$, the free energy profile $\mathrm{F}()=-k B T \ln \mathrm{P}()$ is obtained up to an arbitrary constant, where $k B$ is the Boltzmann constant. The free energy profile has been constructed for each of the 8-rings, which are extracted by the zeoTsites program ${ }^{47} 4^{8}$ taking into account periodic boundary conditions. In our implementation, the pieces of the ( $t$ ) trajectory where the ethene molecule is too far from the ring, i.e. in a non-adjacent cage, are left out, and the counts of all ethene molecules are added to obtain the total number of crossings $C R$ through a ring $R$. If the diffusion is truly an activated 
process and MD sampling was sufficiently long, then $C_{R}$ should relate to the free energy barrier between cage and window.

\section{RESULTS AND DISCUSSION}

A. Diffusion characteristics of ethene and propene

The mean square displacement of both ethene and propene in each of the investigated materials and at three different temperatures $(300,450$ and $600 \mathrm{~K})$ is shown in Fig. 3. It is immediately clear that ethene diffuses through each of the six materials, since a straight slope of the MSD versus time is obtained. For propene this is not the case and in fact the error on the MSD plots is too large to fit a reliable slope. For propene, diffusion itself becomes a rare event at the time scale of the MD simulations and enhanced sampling techniques should be used. Such study is beyond the scope of the present article. Propene accidently hops from one cage to another but not frequently enough to deduce quantitative information (Figs. S24 in Supp. Info.). Given this observation we will mainly focus the rest of the discussion on ethene, where the diffusion has been sampled sufficiently to have statistically significant conclusions.

Visualization of ethene trajectories further reveals that ethene diffuses by a jump process between cages (Fig. S6 in Supp. Info.). The molecule spends most of the time in a cage and occasionally crosses a ring to a neighboring cage. The number of observed ring crossings is sufficiently large to deduce reliable quantitative diffusivities. The ethene diffusivities in each of the materials are listed in Table II. Before discussing these in detail, it is interesting to deduce some qualitative features about the differences in diffusivity in the various materials.

General trends in diffusivity for ethene. The effect of acid sites is dominant: ethene diffusion decreases in all cases when acid sites are introduced. Diffusion is faster in nonacidic materials (SSZIAIPO) than in acidic materials (H-SSZ/SAPO). Furthermore, diffusion increases in general with temperature, which indicates that the hopping process is an activated process for which an empirical Arrhenius law may be fitted (vide infra). The differences induced by composition indicate in general that diffusion in silicoaluminophosphates (AIPO/SAPO materials) is faster than in aluminosilicates (SSZ/H-SSZ materials). Within the set of investigated materials the influence of topology is fairly limited, which is not sur- 
ethene in frameworks without acid sites
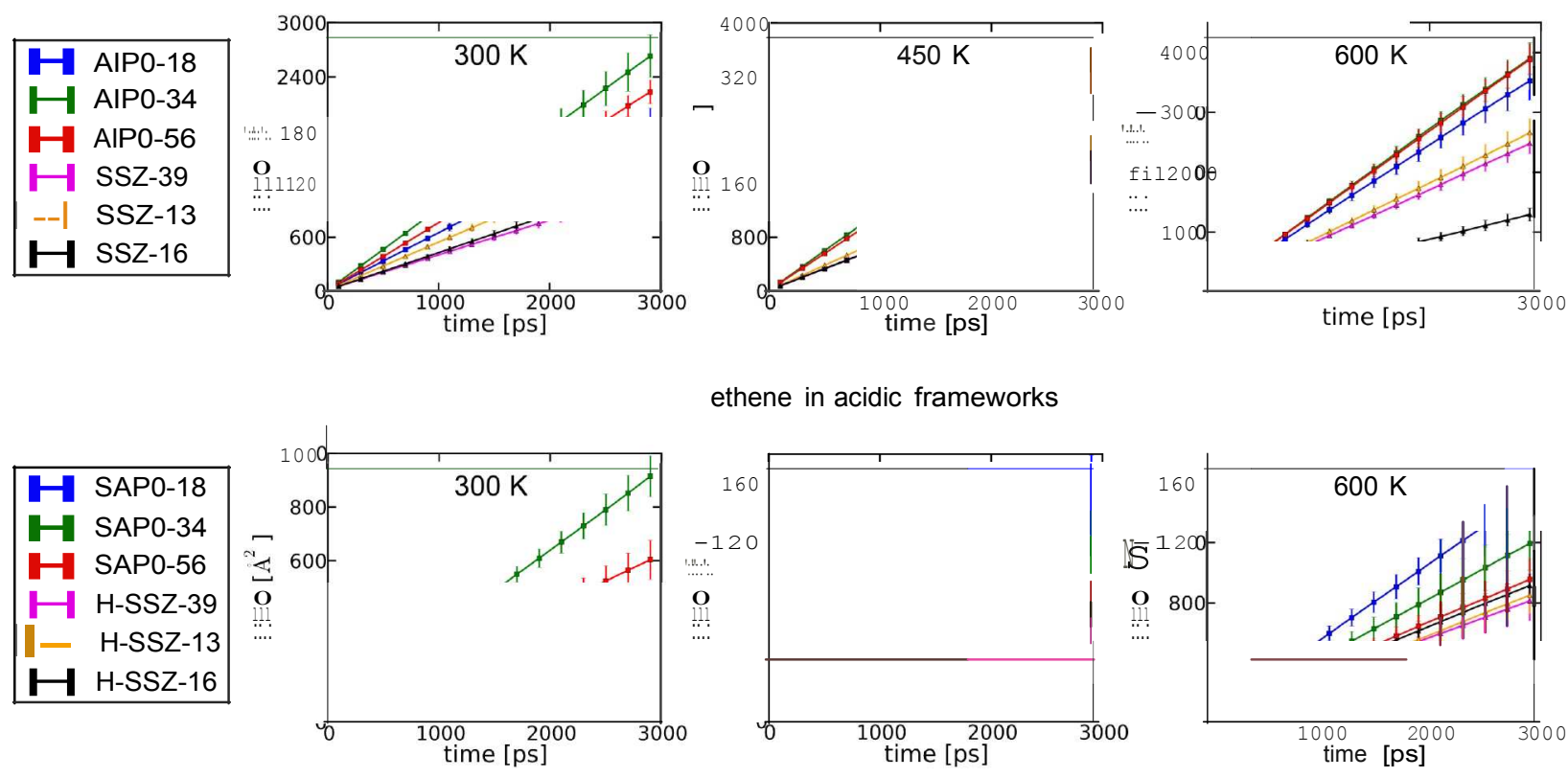

ethene in acidic frameworks
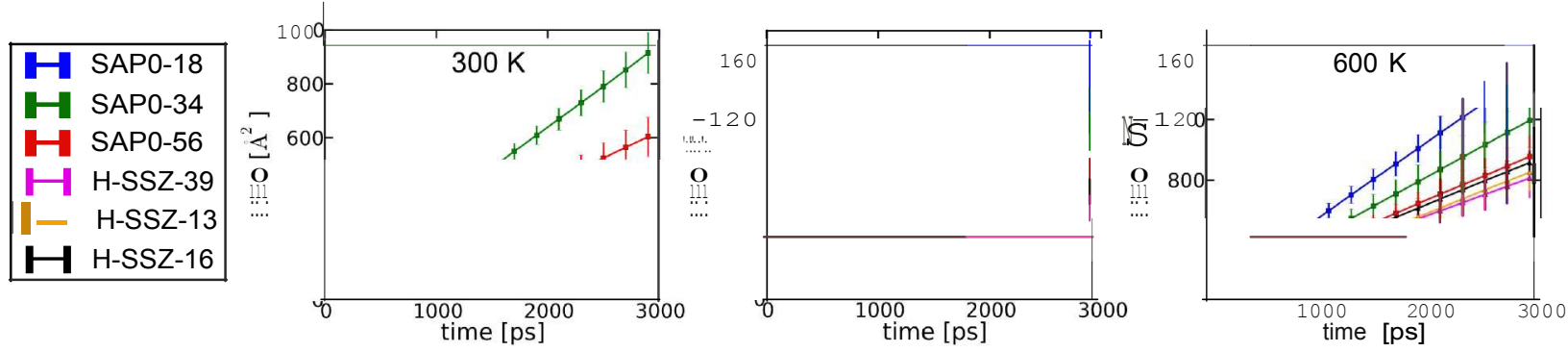

propene in acidic frameworks
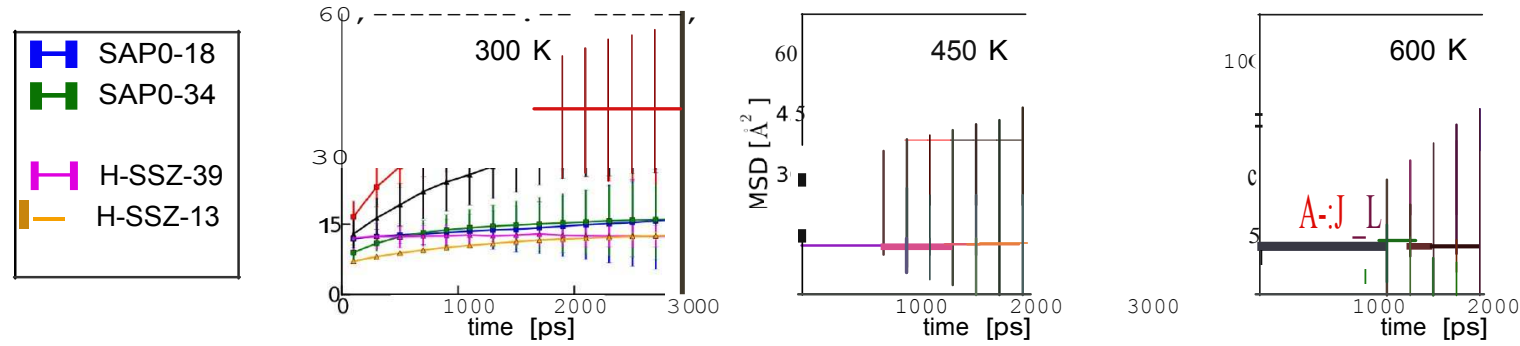

FIG. 3: MSD with error bars $\left[\right.$ in $\left.A^{2}\right]$ of ethene and propene at different temperatures.

prising as AEI, CHA, and AFX frameworks are all 8-rings zeolites. For the AlPO /SAPO materials, diffusion is usually slightly higher in the CHA than in the AFX topology. In these observations, SAP0-18 is an exception, having comparably slow diffusion at low temperature but showing the fastest diffusion at high temperatures. SSZ-16 is also an exception with relatively slower diffusion at high temperature. These exceptions will be shown to originate from framework distortions in Section III D.

Diffusion coefficients and comparison with available literature data. Experimental and simulated data are available for the diffusion coefficients in materials with the CHA topology. First of all we compare our values with simulated diffusion coefficients which are available in the all-Si material with CHA topology, i.e. SSZ-13. Our value for ethene diffusion ranges from 9.0 to $15.210-1 \mathrm{O} \mathrm{m}^{2} / \mathrm{s}$ (300 to $600 \mathrm{~K}$ ) (Table II) in SSZ-13. Wang et al. 
TABLE II: Ethene diffusion coefficient $D$ [in $10^{-10} \mathrm{~m}^{2} / \mathrm{s}$ ] at three temperatures by fitting to MSD curves (Eq. 1), with standard error between brackets.

\begin{tabular}{rrrr}
\hline & $300 \mathrm{~K}$ & $450 \mathrm{~K}$ & $600 \mathrm{~K}$ \\
\cline { 2 - 4 } AIPO-18 & $10.2(1.1)$ & $16.8(1.1)$ & $20.0(1.9)$ \\
AIPO-34 & $15.1(1.1)$ & $20.2(1.6)$ & $23.1(1.8)$ \\
AIPO-56 & $13.0(1.0)$ & $18.0(1.8)$ & $22.7(2.2)$ \\
\hline SSZ-39 & $6.4(0.6)$ & $10.4(0.8)$ & $14.2(1.0)$ \\
SSZ-13 & $9.0(1.0)$ & $12.4(1.0)$ & $15.2(1.4)$ \\
SSZ-16 & $7.0(0.6)$ & $10.5(1.3)$ & $7.4(0.7)$ \\
\hline SAPO-18 & $1.9(0.1)$ & $8.7(1.6)$ & $8.6(1.1)$ \\
SAPO-34 & $5.1(0.4)$ & $6.3(0.7)$ & $6.7(1.1)$ \\
SAPO-56 & $3.3(0.4)$ & $4.7(0.7)$ & $5.3(0.8)$ \\
\hline H-SSZ-39 & $2.0(0.2)$ & $3.4(0.5)$ & $4.6(0.8)$ \\
H-SSZ-13 & $2.2(0.2)$ & $3.8(0.5)$ & $4.8(0.7)$ \\
H-SSZ-16 & $2.4(0.3)$ & $4.2(0.6)$ & $5.1(0.5)$ \\
\hline
\end{tabular}

used the BKS force field ${ }^{70}$ and reported diffusion coefficients for ethene ranging from 9.63 to $14.3710^{-10} \mathrm{~m}^{2} / \mathrm{s}$ from $3 \mathrm{~ns}$ trajectories and 10.44 to $15.3010^{-10} \mathrm{~m}^{2} / \mathrm{s}$ from $18 \mathrm{~ns}$ trajectories $(637$ to $873 \mathrm{~K}){ }^{28}$ With this force field, propene diffusivity was about 20 times lower than ethene diffusivity. ${ }^{28}$ Also Combariza found substantially lower diffusivities for propene in SSZ-13 with a value of 0.52 to $1.010^{-10} \mathrm{~m}^{2} / \mathrm{s}$ originating from simulations with the BKS force field (300 to $600 \mathrm{~K}){ }^{71}$ Overall our values for ethene diffusivity compare relatively well with the reported simulated data. Diffusion data for ethene or propene in the acidic materials are not available in literature yet.

Experimentally, values lie more spread out. Hedin et al. performed PFG NMR experiments with all-Si-CHA (SSZ-13) at $301 \mathrm{~K}$ and 1 bar, corresponding to 1.4 ethene molecules per cage, and observed an ethene diffusivity of $0.031( \pm 0.014) 10^{-10} \mathrm{~m}^{2} / \mathrm{s}$. $^{72}$ This value lies below the computed ones, but the sensitivity of this type of experiments specifically to bulk diffusivities and the reproducibility with various samples have been questioned. ${ }^{21}$ Olson et al. found a value of $0.001110^{-10} \mathrm{~m}^{2} / \mathrm{s}$ for propene in all-Si CHA at $353 \mathrm{~K}$ with an activation bar- 
rier of $10 \mathrm{~kJ} / \mathrm{mol}$ from an adsorption experiment. ${ }^{73}$ They report that ethene diffuses about 28 times faster than propene at that temperature, and 80 times faster at $303 \mathrm{~K} .{ }^{73}$ Ruthven and Reyes used a similar adsorption experiment to derive various diffusion coefficients, among which those of ethene and propene in the acidic material SAPO-34. From these experiments it was derived that ethene and propene have diffusivities of 0.0015 and $0.000910^{-10} \mathrm{~m}^{2} / \mathrm{s}$ in SAPO-34 at $323 \mathrm{~K}$ and activation barriers 82 and $97 \mathrm{~kJ} / \mathrm{mol}$, respectively. ${ }^{74}$ The SAPO-34 material has also been investigated experimentally by Dai et al. ${ }^{23}$ They derived PFG NMR ethene diffusivities of 0.13 to $0.1610^{-10} \mathrm{~m}^{2} / \mathrm{s}$ for loadings of 1 to 3 molecules/cage at $295 \mathrm{~K}$, which is one order of magnitude lower than in our simulations, and found an activation barrier of $4.2 \mathrm{~kJ} / \mathrm{mol}$ in the temperature range $303-353 \mathrm{~K}$. In summary, the values vary largely between simulations, experiments and experimental techniques. Rather than focussing on quantitative reproduction of some observed measurements, the primary goal of this paper is to reveal the molecular factors that control the diffusivity.

The increase in ethene diffusivity with temperature suggests that the diffusion is an activated process. An Arrhenius plot may be fit to the $D$ values at the three temperatures. The values for the derived Arrhenius parameters are taken up in the Supp. Info. (Table S4, Fig. S5). In general, preexponential factors are highest for the AIPO materials and nonacidic zeolites, indicating lower intrinsic diffusivity for acidic materials. On the other hand, the activation energies are higher for zeolites, meaning that diffusion in zeolites can be enhanced more easily with temperature than in SAPOs, which explains the observed higher diffusivities in the 300 to $600 \mathrm{~K}$ range. For the materials SAPO-18 and SSZ-16, it is not possible to fit Arrhenius parameters reliably, because the observed variations in diffusivity with temperature is caused by framework distortions, as will be explained later in Sec. IIID.

Diffusion coefficients in relation to the material's flexibility. Since our simulations use a flexible framework, and 8-rings are tight-fitting windows for the hydrocarbon diffusion, it is tested whether the diffusivities correlate to the flexibility of the material. A material's overall flexibility is here assessed by the root mean square fluctuation (RMSF) per T-atom, i.e. the square root of the time-averaged squared deviations of the framework atom positions with respect to the time-averaged structure, as displayed in Figs. S7 and S8 in the Supp. Inf. For a given material, flexibility and diffusivity go hand in hand: an increase in temperature is systematically accompanied by an increase in both flexibility and diffusion coefficient. However, at a given temperature, trends in flexibility between the materials do 
not follow trends in diffusion coefficients. A flexibility parameter like RMSF per T-atom cannot differentiate in diffusivity between materials. This observation lies in line with studies in literature that investigate the effect of a rigid framework. ${ }^{33,35,44}$ As mentioned in the introduction, there is no general rule that predicts the effect of a flexible framework simulation on the diffusion compared to a rigid framework simulation: including flexibility may enhance or lower diffusivities depending on the considered framework-adsorbate system.

It should be stressed that diffusivities and flexibility are global material-adsorbate parameters. The diffusivities and overall flexibility as described here are global parameters which incorporate very diverse contributions from the individual rings. To understand the molecular origin for the diffusion behavior, it is important to perform a detailed analysis of the individual 8-rings. It will become clear in the next sections that individual rings can have significantly different free energy barriers for ring crossing.

\section{B. Ring character and free energy barriers/crossings}

In a previous paper by one of the authors it was found that AFX and AEI topologies respond structurally to adsorption of propane molecules. ${ }^{29}$ At low temperature, the materials loose some symmetry due to ring deformations. At higher loading and at higher temperatures, the average structure is again more symmetric. This flexible response of the material has a large effect on the diffusion even if the deformations seem small, because the 8-rings are tight-fitting for diffusion of ethene and propene (Fig. 1). A flexibility parameter like RMSF per T-atom is too global to differentiate in diffusivity between materials. Therefore a detailed analysis is here presented of the individual ring properties.

The purpose of the analysis is to asses which factors affect the number of crossing molecules through a given 8-ring R. First of all the various 8-rings encountered in each of the materials are classified according to two factors, namely the ring topology and ring composition as schematically shown in Fig. 4. We will refer to the combination of ring topology and ring composition as the ring character.

The first factor is the local ring topology (Fig. 4), i.e. which two cages are connected by the ring, and which other adjoining rings are attached to the ring. A schematic view of ring topology is presented in Fig. 4. In CHA materials, all cages are identical, and all 8-rings are topologically equivalent. In AEI materials, all cages are identical as well, but not all 8-rings 
Inventory rings

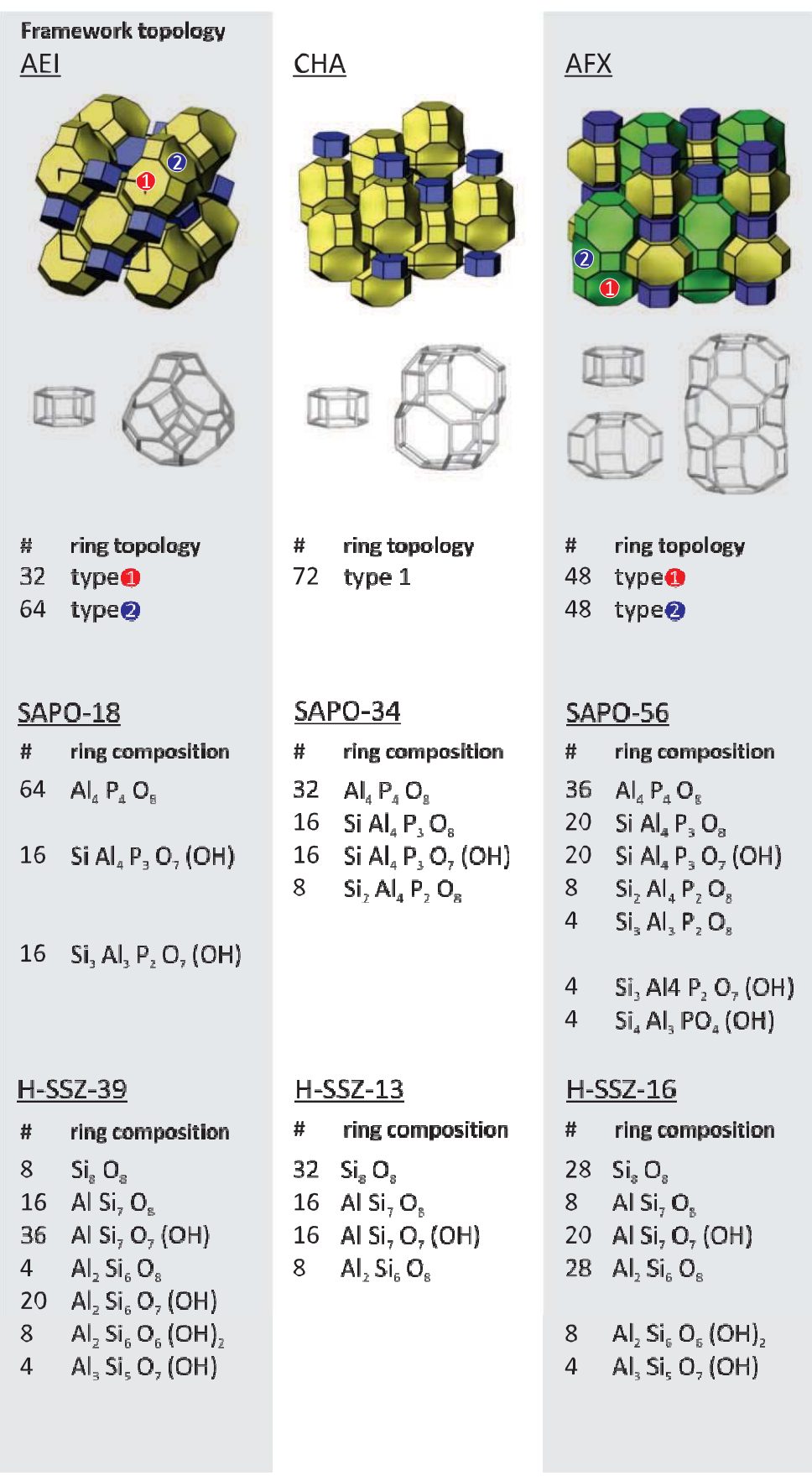

FIG. 4: Inventory of rings according to ring topology and ring composition (ring character). Top part of figure is adapted from IZA database. ${ }^{2}$

are topologically equivalent, as the adjoining rings differ. One third of the rings are of type 1 and two thirds of the rings are of type 2. AFX materials have large cages and small cages. In AFX, ring type 1 connects two large cages, while ring type 2 connects a small and a large 
cage.

The second factor is rzng composition. The acid sites are distributed over the framework, and some rings may contain protons and/or acidic sites, creating an inhomogeneous composition distribution of the rings. It was already observed in the MSD (Fig. 3) that the presence of an acidic proton decreases the overall diffusivity of ethene. This might be ascribed to the reduction of available space, or specific interactions with the adsorbates or other structural variations in the ring. Indeed, the chemical variety may cause deviations from the pure-silica Si-0 bond lengths and O-Si-O angles, leading to structural distortions. Crossing through a specific ring is thus expected to depend on the ring composition. An inventory of ring compositions is also given in Fig. 4.
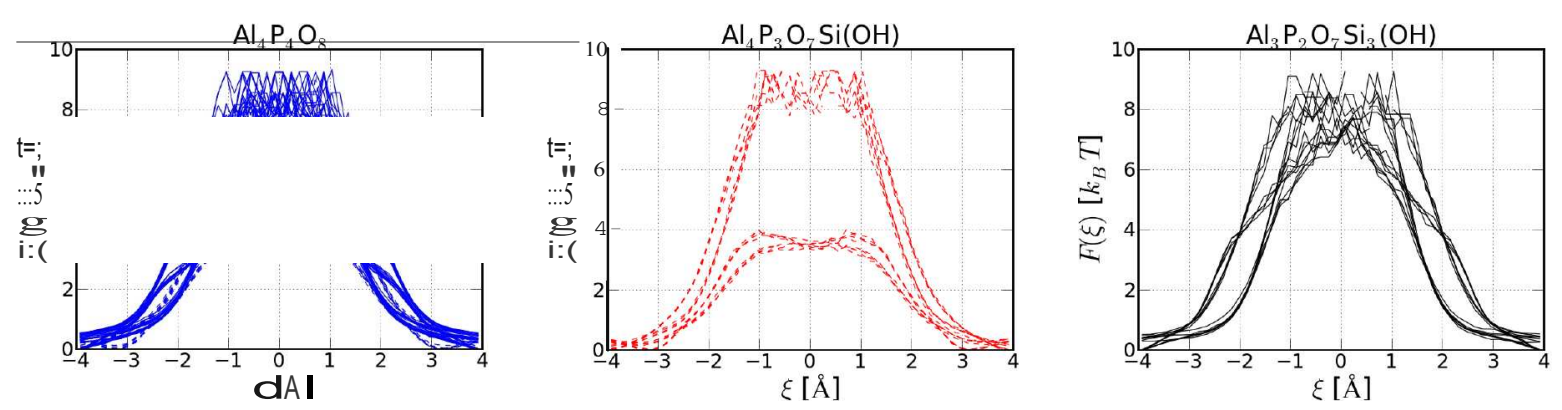

FIG. 5: Free energy profile $\mathrm{F}($ ) (in units $k B T$ ) for ethene passing through each of the rings of SAP0-18 at $450 \mathrm{~K}$. One line per ring; line may appear broken when a histogram bin is empty (Section IIF). SAP0-18 has three ring compositions: Al4P40s (blue), A14P307Si(OH) (red) and $\mathrm{AhP207Si3}(\mathrm{OH})$ (black). It has two ring types: type 1 (dashed lines) and type 2 (solid lines).

The free energy profiles $F($ ) (Fig. 5) for each of the nngs are constructed by histogramming the position coordinate of ethene with respect to the ring (definition in Section II F); each profile is shifted to have its minimum at zero. Remarkably, these free energy profiles cluster into groups. For instance, consider the rings of type 1 and composition A14P3O7Si(OH) in SAP0-18 at $450 \mathrm{~K}$, shown in the middle pane of Fig. 5. These rings do not have the same free energy profile in spite of having the same ring character. Surprisingly, two distinct clusters of free energy profiles are observed. They differ mainly in their barrier height $(4 k B T$ versus $9 k B T)$, meaning that individual rings may have significantly different activation barriers for the hopping process. Such clustering of free energy profiles is less pronounced in the H-SSZ-39 and H-SSZ-13 materials (other materials may be found 


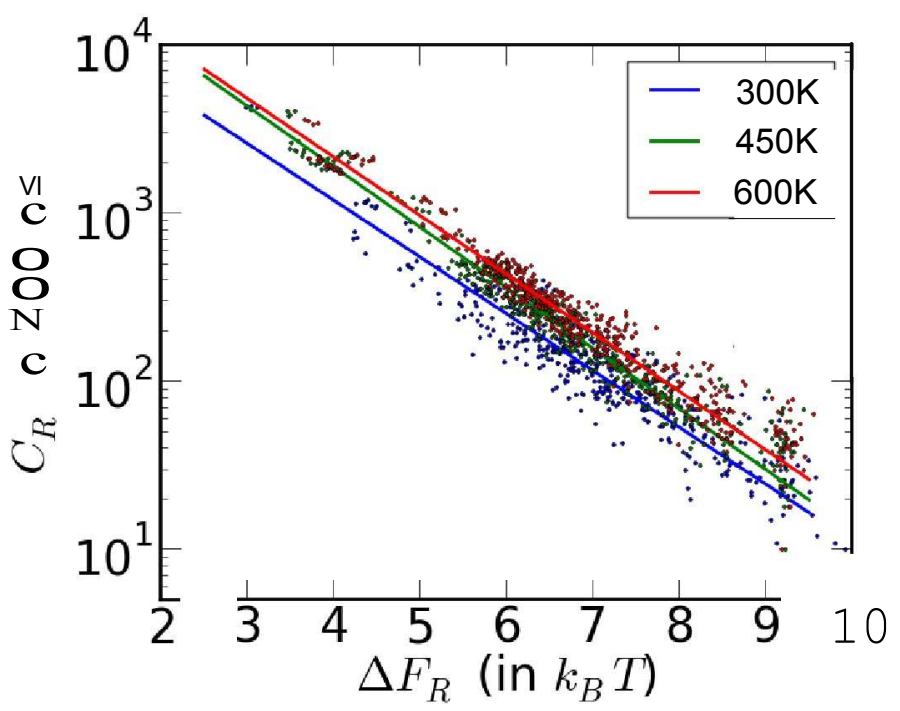

FIG. 6: Correlation between observed ring crossing $C R$ per $200 \mathrm{~ns}$ and free energy barrier $F R$ (in units $k B T$ ). Each dot represents a ring in one of the six acidic materials. Lines are least square fits (Eq. 3) at three temperatures.

in Figs. S9 in Supp. Inf.).

We have also computed the number of crossings $C R$ of ethene molecules through each ring $R$. Very diverse crossing counts are found, confirming that not all rings are equivalent. Some 'slow' rings have about five or even zero crossings in our simulated trajectory, while other 'fast' rings have up to 4000 observed crossings in the same MD trajectory. Correlating the crossing count $C R$ of each ring with its free energy barrier height $F R$ is a verification that the diffusion is an activated process. The Boltzmann factor $\exp (-F R /(k B T))$ is the probability ratio of ethene being at the top of the barrier (0) and ethene being in the cage. If the diffusion is activated and in thermodynamic equilibrium, $F R$ should therefore relate to the ring crossings,

$$
C_{R}=C_{R}^{0} e^{-\Delta F_{R} /\left(k_{B} T\right)}
$$

Combining data of all rings of all six acidic materials at all three temperatures, Fig. 6 provides a confirmation of the relation described in Eq. 3. The number of ring crossings $C R$ covers two orders of magnitude, but still a line may be fit on a logarithmic plot which confirms that ethene diffusion is indeed an activated process. The prefactor $C$ may be interpreted as the mobility in absence of any barrier for diffusion, so-called free diffusion.

The large diversity in ring crossings (two orders of magnitude) observed in this work has 


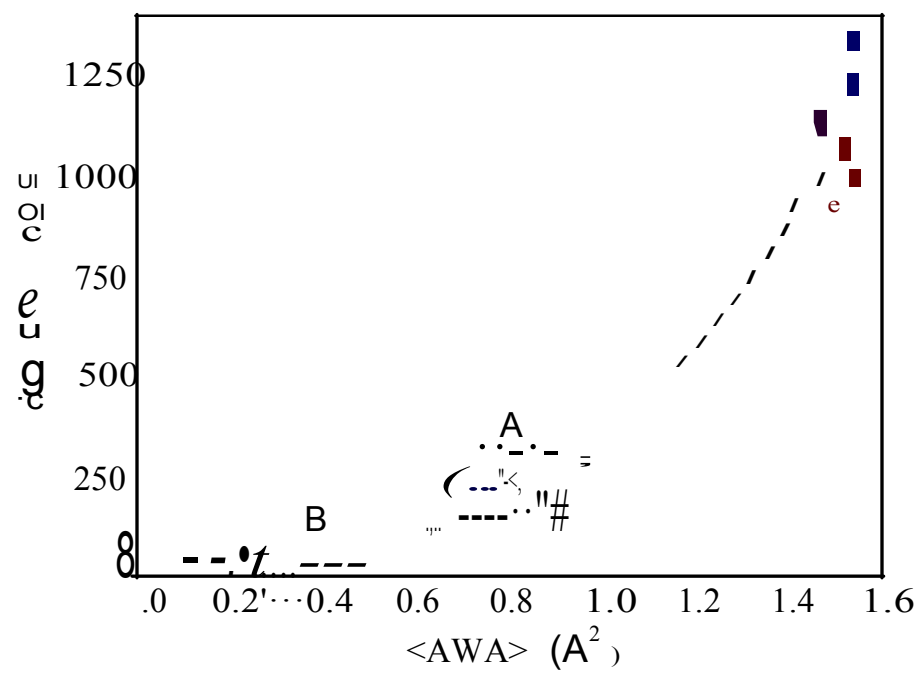

FIG. 7: Number of crossings versus (dmin) (top) and (AWA) (bottom) for SAP0-18 in a lOOns trajectory at 450K. Symbol color according to ring composition: A14P40s (blue), Al4P307Si(OH) (red) and Al3P207Si3(0H) (black). Symbol shape according to ring topology: type 1 (circle) and type 2 (diamond). The dashed line represents a fitted exponential function.

not been reported in literature before. The activation energy has been linked to the ring dimension, usually quantified by the average minimum diagonal O-O distance (dmin)- $-33,34,6475$ The larger the ring diameter, the more space is available for ethene to cross the window. To validate whether the ring diameter is a proper molecular descriptor for the number of ring crossing, we plot the crossings $C R$ versus the average minimum diagonal 0-0 distance 
$\left(d_{\text {min }}\right)$ in Fig. 7 for the SAPO-18 material at $450 \mathrm{~K}$. It is remarkable that the crossing counts cluster into groups. Although clustering is often due to differences in ring character, this is not always the case. For example, the two clusters $A$ and $B$ in Fig. 7 have identical ring character (indicated by blue diamonds), but their crossing counts are different. This means that the two factors - ring topology and ring composition - are valuable descriptors, but are unable to fully capture how slow/fast the diffusion would occur. Moreover the minimum diagonal O-O distance $\left(\mathrm{d}_{\min }\right)$ is also not able to discriminate the variations in ring diffusivity observed between clusters $A$ and $B$. In the next Section III C we will introduce a new geometrical descriptor that can explain the clustering.

\section{A new molecular descriptor for ring crossings}

In Fig. 7, many more crossings are observed for cluster A versus cluster B, even though the computed $\left(d_{\min }\right)$ is smaller. The first and second factor (ring topology, ring composition) cannot explain this difference, and therefore the rings' structures are compared in detail to reveal a third factor. In an attempt to find a molecular descriptor which correlates well with the observed ring crossings, a number of alternative ring descriptors were computed. The details may be found in the Supp. Info (see Figs. S10-12). Some describe ring size (average ring area computed as the area of the fitted ellipse of Section II F, average maximum distance $\left(d_{\max }\right)$ between opposite T-site atoms). Some describe ring deformation in the plane (average ratio $\left(d_{\max } / d_{\min }\right)$, average eccentricity of the fitted ellipse), or ring deformation out of the plane (average boat, chair and total puckering amplitudes). Others describe ring flexibility (standard deviation on $d_{\min }$, probability $P\left[d_{\min }>d_{t}\right]$ of having a distance $d_{\min }$ above $a$ certain chosen treshold value $d_{t}$ ).

None of these computed structural parameters can explain all trends in ring crossings. Visualization of clusters $A$ and $B$ rings (bottom panel in Fig. 8) shows that the displacement of some of the ring atoms towards the inside of the ring seriously reduces the available space for ethene to pass. As a new parameter that can capture this effect, we propose the accessible window area (AWA) as a structural descriptor. AWA is inspired by the solvent accessible surface area that is commonly used in implicit solvation models of proteins and small molecules. ${ }^{76}$ The ethene AWA of a ring is the free space available to an ethene molecule when placed inside a ring. It is computed by rolling a disk with the size of an ethene molecule 
along the inner edges of the ring atoms (details in Supp. Info.). AWA is sensitive to the inward bending of ring atoms in cluster $\mathrm{B}$, whereas the more rudimentary parameter (dmin) was not.
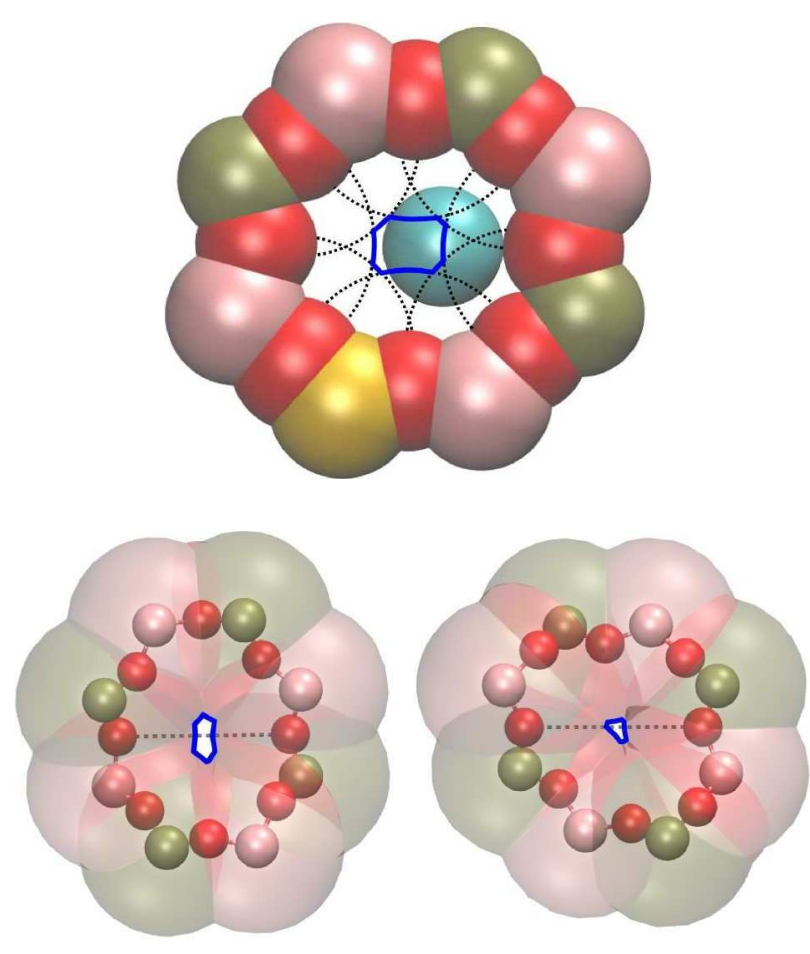

cluster $A$ (faster)

dmin $=6.03 \mathrm{~A}$

cluster B (slower)

$A W A=0.85 \mathrm{~N}$

$\mathrm{dmin}=6.15 \mathrm{~A}$

AWA $=0.39 \mathrm{~N}$

FIG. 8: (Top) Definition and computation of accessible window area (AWA) of 8-ring. The ethene molecule is represented by a blue colored sphere and rolls over the surface formed by the vdW radii of the oxygen atoms. The trajectory of the ethene sphere center (blue line)

delimits the accessible window surface, whose area is computed with triangulation.

(Bottom) The geometry of two specific rings in SAP0-18, representing clusters A and B in Fig. 7, with indicated dmin and AWA parameters. In the slower ring (right), the available space for ethene is much smaller despite the larger dmin value, than in the faster ring (left), because some ring atoms are bended towards the inside of the ring.

The average AWA ((AWA)), and its correlation with the ring crossing count is considerably better than that of (dmin), as demonstrated in Fig. 7, bottom panel, and Fig. S13. Coloring of individual rings in Fig. 9 also shows a remarkable similarity between (AWA) and the ring crossings $C R$ for all six materials. AWA measures the available space and its high correlation with the ring passages is therefore not surprising. The free energy barriers 

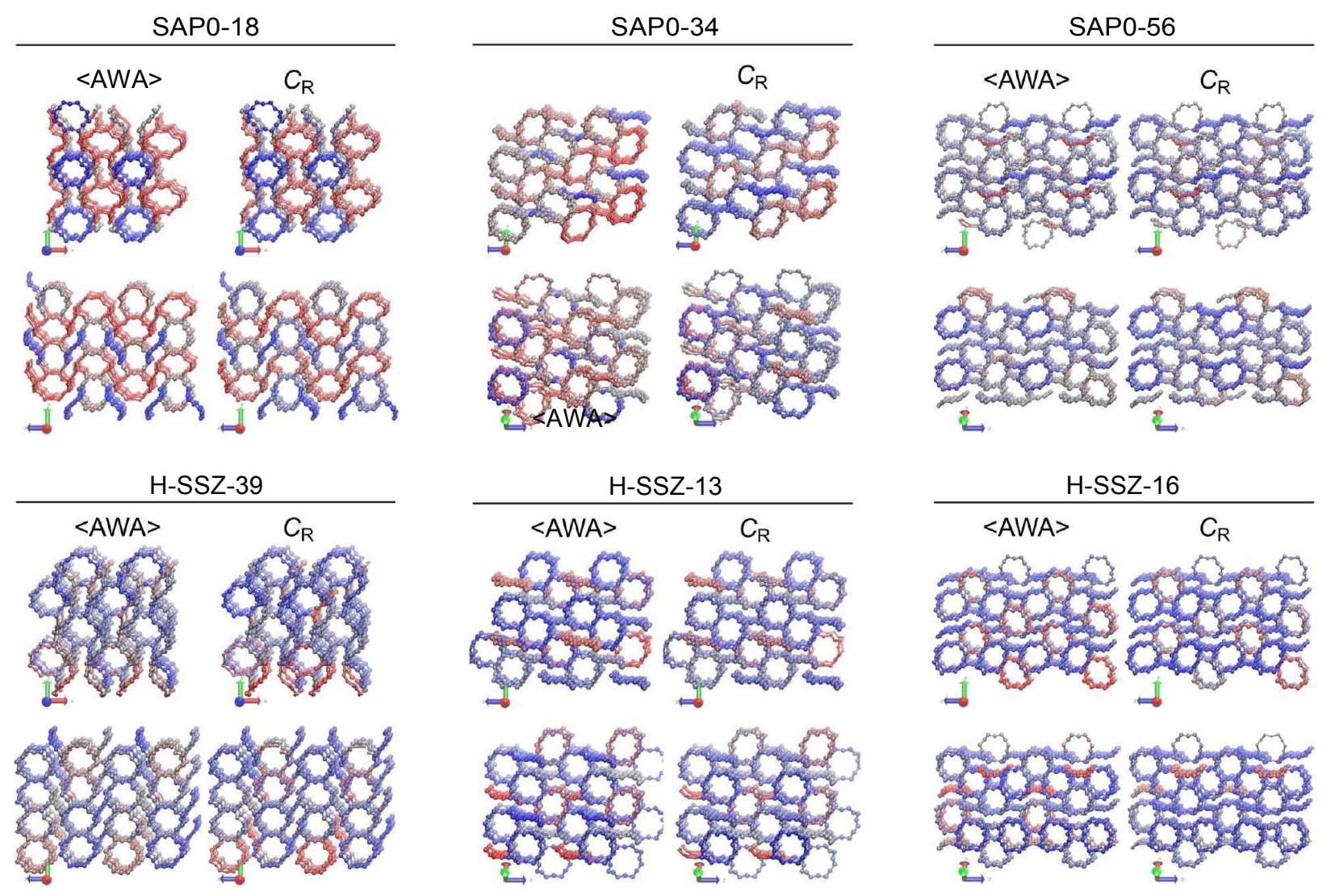

low

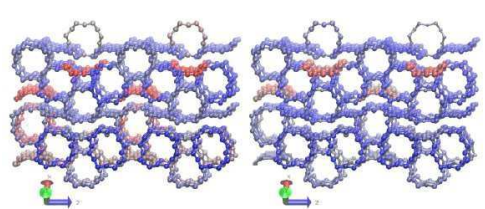

high

FIG. 9: AWA correlates with ring crossings in the six acidic materials. Rings are colored according to their structural parameter (AWA) (left) and their ring crossings $C R$ (right), at $450 \mathrm{~K}$. Each structure is shown from two different viewing angles as indicated by the axes ( $x, y$, and $z$ axes are colored red, green, and blue, respectively). The color gradient (redgray-blue) corresponds with increasing values of (AWA) (linear scale) and ring crossings ( $\log$ scale).

in Fig. 5 are thus closely related to the structural parameter AWA. AWA comprises both enthalpic and entropic contributions to the free energy. When AWA decreases, the ring is tighter which may lead to stronger interactions between ethene and ring atoms, hence an enthalpic effect. Moreover, a tighter ring offers less space to ethene to pass, which is clearly an entropic effect.

The performance of AWA is compared to some of the previously mentioned structural ring parameters, e.g. dmin and the dmax/dmin. The latter is a measure for the eccentricity if the ring were to be an ellipse. The Spearman correlation coefficient between the structural 


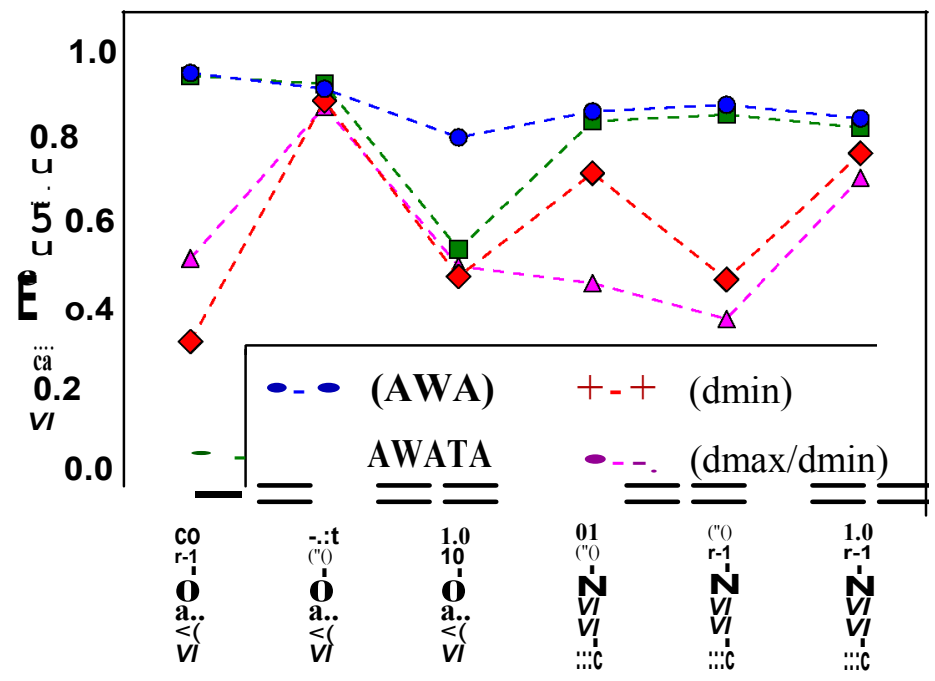

FIG. 10: Spearman correlation coefficient between structural ring parameter and ring crossings are plotted for the six acidic materials at $450 \mathrm{~K}$. The Spearman correlation coefficient is chosen, because it is sensitive to the ordering of data rather than to the value and is thus suitable for the extended data range of crossing counts. (.) denotes an average structural ring parameter; AWATA is AWA computed for the time-averaged structure; dmax is maximum diagonal $\mathbf{O}-\mathbf{O}$ distance.

parameter and ring crossings is shown Fig. 10. The higher the correlation, the better a structural parameter can predict ring crossings. Our (AWA) parameter outperforms other structural parameters in all six acidic materials, suggesting that (AWA) is a considerably improved predictor, and is much better at capturing the ring structure in a single parameter.

The analysis as performed here, clearly shows that even for rings having similar topology and similar composition, individual ring crossings are not homogeneously distributed over the lattice.

\section{Framework dynamics}

A longstanding question is how framework flexibility affects diffusion in the investigated systems. For SAP0-18 an abrupt increase in diffusion coefficient $D$ was noted between $300 \mathrm{~K}$ and $450 \mathrm{~K}$ in Table II, and furthermore for this system it was not possible to deduce Arrhenius parameters to describe the diffusivity. Having introduced the AWA molecular 


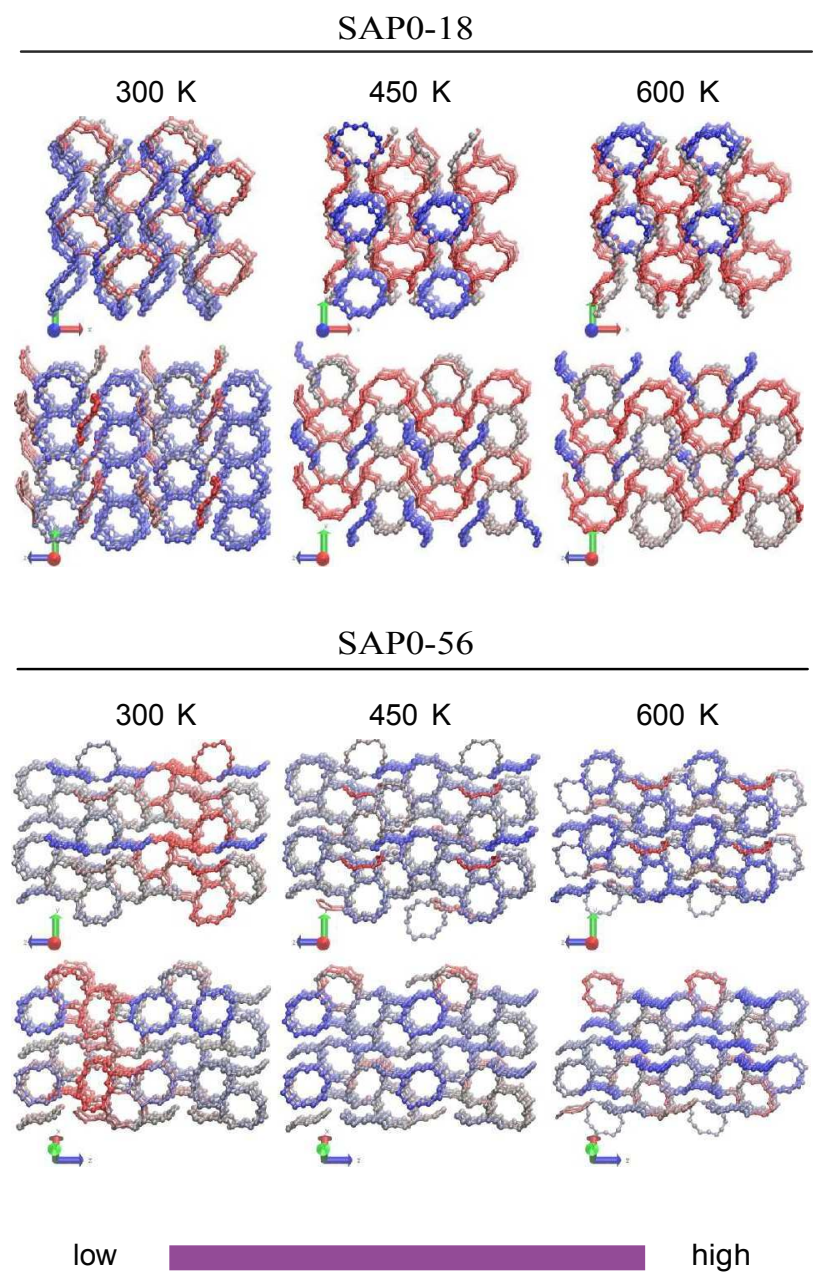

FIG. 11: Framework dynamics: deformations due to temperature. Rings are colored according to their structural parameter (AWA); the color gradient (red-gray-blue) corresponds with increasing values of (AWA) (linear scale). SAP0-18 (top) undergoes a transition between $300 \mathrm{~K}$ and $450 \mathrm{~K}$. SAP0-56 (bottom) at $450 \mathrm{~K}$ switches seven times between its $300 \mathrm{~K}$ and $600 \mathrm{~K}$ states in the course of a $100 \mathrm{~ns}$ trajectory. Each structure is shown from two different viewing angles as indicated by the axes $(x, y$, and $z$ axes are colored red, green, and blue, respectively).

descriptor, it becomes clear that the abrupt increase in $D$ is due to framework deformations. Fig. 11 shows that the rings in SAP0-18 deform significantly in terms of temperature, as is evident in their accessible window area. The observed changes in AWA lead in turn to significant changes in crossing counts and thus overall a higher diffusion coefficient. The increasing $D$ value at $450 \mathrm{~K}$ is thus not a pure consequence of increasing ethene mobility and ring flexibility with increasing temperature, but also due to a structural deformation. 
Also for SAPO-56 a structural deformation between $300 \mathrm{~K}$ and $600 \mathrm{~K}$ (Fig. 11) is noticed. At lower temperatures $(300 \mathrm{~K})$ two bands of narrow and wide rings parallel to the $x y$-plane are present. At higher temperature $(600 \mathrm{~K})$, wide rings predominate in the direction normal to the $\mathrm{x}$-axis, while narrow rings are mostly found normal to the y-axis. At $450 \mathrm{~K}$, the SAPO-56 framework is in equilibrium between the two states. In our 100 ns trajectory at $450 \mathrm{~K}$, seven transitions between the two states are observed, and both states are sampled about equally. Consequently, AWA has a bimodal distribution, and the signature of (AWA) at $450 \mathrm{~K}$ is intermediate between the $300 \mathrm{~K}$ and $600 \mathrm{~K}$ figures in Fig. 11.

Our averaged AWA descriptor ((AWA)) correlates well with the ring crossings as observed in Fig. 10. To facilitate the computation of structural parameters, one could opt to calculate the AWA once at the time-averaged (TA) structure, which is obtained by taking the time average of the framework coordinates over the whole trajectory. The correlation of this parameter, denoted by AWA ${ }^{T A}$, with the number of ring crossings is also shown in Fig. 10. In most of the cases, AWA $^{T A}$ captures the ring crossings sufficiently, except in those cases where framework deformations are large, or have a bimodal distribution, as is the case for SAPO-56 at $450 \mathrm{~K}$. When a two-state dynamics is observed of the system, the time-averaged structure is not able to capture the behavior along the whole trajectory and at least two structures should be included in the evaluation of the AWA parameter.

\section{CONCLUSIONS}

In this paper, we investigated diffusion of ethene and propene in a series of 8-ring zeolites to assess the influence of topology, acidity and flexibility on the diffusion in confined zeolites and zeotype materials. The study is inspired by the knowledge that diffusion of product species is important to determine the selectivity in the methanol to olefin (MTO) process. We have investigated the AEI, CHA and AFX topology which are all relevant for the MTO process. In each of the cases we have considered the all silica and aluminophosphate versions of the isostructural materials. Furthermore the influence of inclusion of acid sites is investigated. To properly assess the influence of the flexibility of the material all MD simulations were performed using a flexible force field, which was designed for the problem at hand.

First of all, some general features concerning the diffusivity were observed. As expected, ethene diffuses much more easily than propene, and only for ethene it is meaningful to derive 
quantitative diffusion coefficients based on MD runs of 100-200 ns. Inclusion of acid sites in the material yields a slower diffusion, and diffusion is in general faster in AIPO/SAPO materials than in SSZ/H-SSZ materials. In general diffusion of ethene is an activated process, meaning that the molecule spends most of the time within a cage and occasionally hops from one cage to the other. To jump from one cage to the other a free energy has to be overcome, which may differ quite substantially depending on the nature of the ring. Apparently, some rings have identical characteristics such as ring topology and ring composition but still show different ring crossings.

A new molecular descriptor had to be introduced to find a good correlation with the number of ring crossings, namely the accessible window area (AWA), which measures the free space available for ethene molecules. The concept is inspired by the accessible surface area that is often used in implicit solvation models. The free energy barriers for the individual rings can be correlated with the AWA parameter, which may be assessed along the MD trajectories.

For some materials such as SAPO-18, framework dynamics contributed to large changes in the diffusivity in terms of temperature. Indeed at lower temperature, SAPO-18 shows low diffusivity whereas at higher temperatures structural transformations at the level of the individual rings have taken place which contribute to a larger diffusivity. The observed effects can also be explained in terms of the newly introduced AWA descriptor. Summarizing, this study has shown that overall observed diffusivity is determined by molecular characteristics of individual rings for which proper descriptors need to be used. The new descriptor as introduced here should be applicable to other materials and provides a good basis for studying the molecular factors controlling diffusion.

\section{ACKNOWLEDGEMENT}

The computational resources and services used were provided by Ghent University (Stevin Supercomputer Infrastructure). Funding was received from the Research Board of Ghent University (BOF), the Foundation of Scientific Research - Flanders (FWO), and BELSPO in the frame of IAP/7/05. V. Van Speybroeck acknowledges funding from the European Research Council under the European Community's Seventh Framework Programme (FP7(2007-2013) ERC grant agreement number 240483), and from the European Union's 
Horizon 2020 research and innovation programme (consolidator ERC grant agreement No 647755 - DYNPOR (2015-2020)). G. Sastre thanks the Spanish government for the provision of Severo Ochoa project (SEV 2012-0267) and SGAI-CSIC for computing time.

1 V. Van Speybroeck, K. Hemelsoet, L. Joos, M. Waroquier, R. G. Bell, and C. R. A. Catlow. Advances In Theory And Their Application Within The Field Of Zeolite Chemistry. Chem. Soc. Rev., pages -, 2015.

2 IZA database. http://www. iza-structure.org/databases. Accessed: 2015-08-22.

3 C. Baerlocher, L. B. McCusker, and D. H. Olson. Atlas of Zeolite Framework Types, 6th Ed., Elsevier, Amsterdam, 2007.

4 A. Corma. From Microporous To Mesoporous Molecular Sieve Materials And Their Use In Catalysis.Chem. Rev., 97(6):2373-2420, 1997.

5 D. E. De Vos, M. Dams, B. F. Sels, and P. A. Jacobs. Ordered Mesoporous And Microporous Molecular Sieves Functionalized With Transition Metal Complexes As Catalysts For Selective Organic Transformations. Chem. Rev., 102(10):3615-3640, 2002.

6 W. Vermeiren and J.-P. Gilson. Impact Of Zeolites On The Petroleum And Petrochemical Industry. Topics In Catalysis, 52(9):1131-1161, 2009.

7 P. A. Jacobs, M. Dusselier, and B. F. Sels. Will Zeolite-Based Catalysis Be As Relevant In Future Biorefineries As In Crude Oil Refineries? Angew. Chem. Int. Ed., 53(33):8621-8626, 2014.

8 A. Primo and H. Garcia. Zeolites As Catalysts In Oil Refining. Chem. Soc. Rev., 43(22):7548$7561,2014$.

9 M. Stöcker. Methanol-To-Hydrocarbons: Catalytic Materials And Their Behavior. Micropor. Mesopor. Mater., 29:3, 1999.

10 U. Olsbye, S. Svelle, M. Bjørgen, P. Beato, T. V. W. Janssens, F. Joensen, S. Bordiga, and K. P. Lillerud. Conversion Of Methanol To Hydrocarbons: How Zeolite Cavity And Pore Size Controls Product Selectivity. Angew. Chem. Int. Ed., 51:5810-5831, 2012.

11 K. Hemelsoet, J. Van der Mynsbrugge, K. De Wispelaere, M. Waroquier, and V. Van Speybroeck. Unraveling The Reaction Mechanisms Governing Methanol-To-Olefins Catalysis By 
Theory And Experiment. ChemPhysChem, 14:1526, 2013.

12 J. F. Haw, W. Song, D. M. Marcus, and J. B. Nicholas. The Mechanism Of Methanol To Hydrocarbon Catalysis. Acc. Chem. Res., 36:317-326, 2003.

13 F. Bleken, M. Bjorgen, L. Palumbo, S. Bordiga, S. Svelle, K. P. Lillerud, and U. Olsbye. The Effect of Acid Strength On the Conversion Of Methanol to Olefins Over Acidic Microporous Catalysts With the CHA Topology. Topics in Catal., 52(3):218-228, 2009.

14 B. P. C. Hereijgers, F. Bleken, M. H. Nilsen, S. Svelle, K.-P. Lillerud, M. Bjrgen, B. M. Weckhuysen, and U. Olsbye. Product Shape Selectivity Dominates the Methanol-to-Olefins (Mto)

Reaction Over H-SAPO-34 Catalysts. J. Catal., 264(1):77-87, 2009.

15 I. M. Dahl and S. Kolboe. On the Reaction-Mechanism for Propene Formation in the MTO Reaction Over SAPO-34. Catal. Lett., 20:329-336, 1993.

16 I. M. Dahl and S. Kolboe. On the Reaction Mechanism for Hydrocarbon Formation from Methanol Over SAPO-34: 1. Isotopic Labeling Studies of the Co-Reaction Of Ethene and Methanol. J. Catal.,149:458-464, 1994.

17 I. M. Dahl and S. Kolboe. On the Reaction Mechanism for Hydrocarbon Formation from Methanol Over SAPO-34: 2. Isotopic Labeling Studies of the Co-Reaction Of Propene and Methanol. J. Catal., 161(1):304-309, 1996.

18 D. Lesthaeghe, V. Van Speybroeck, G. B. Marin, and M. Waroquier. Understanding the Failure of Direct Cc Coupling in the Zeolite-Catalyzed Methanol-to-Olefin Process. Angew. Chem. Int. Ed., 45(11):1714-1719, 2006.

19 D. Lesthaeghe, V. Van Speybroeck, G. B. Marin, and M. Waroquier. The Rise And Fall Of Direct Mechanisms in Methanol-to-Olefin Catalysis: An Overview of Theoretical Contributions. Ind. Eng. Chem. Res., 46(26):8832-8838, 2007.

20 V. Van Speybroeck, K. De Wispelaere, J. Van der Mynsbrugge, M. Vandichel, K. Hemelsoet, and M. Waroquier. First Principle Chemical Kinetics in Zeolites: The Methanol-to-Olefin Process As a Case Study. Chem. Soc. Rev., 43(21):7326-7357, 2014.

21 J. Kärger. Comment on "PFG NMR Self-Diffusion of Small Hydrocarbons in High Silica DDR, CHA And LTA Structures" [micropor. mesopor. mater. 109 (2008) 327]. Micropor. Mesopor. Mat., 116:715-717, 2008.

22 A. Vidoni and D. M. Ruthven. Experimental Self-Diffusion Coefficients of $\mathrm{C} 2 \mathrm{H} 6$ and $\mathrm{C} 2 \mathrm{H} 4$ in DDR. Ind. Eng. Chem. Res., 51:1383, 2012. 
23 W. Dai, M. Scheibe, L. Li, N. Guan, and M. Hunger. Effect of the Methanol-to-Olefin Conversion on the Pfg Nmr Self-Diffusivities of Ethane and Ethene In Large-Crystalline SAPO-34. J. Phys. Chem. C, 116(3):2469-2476, 2012.

24 J. Kärger and D. M. Ruthven. Diffusion in Zeolites and Other Microporous Solids, page 605. John Wiley, New York, USA, 1992.

25 J. Kärger. Transport Phenomena in Nanoporous Materials. ChemPhysChem, 16:24-51, 2015.

26 B. Smit and T. L. M. Maesen. Molecular Simulations Of Zeolites: Adsorption, Diffusion, and Shape Selectivity. Chem. Rev., 108:4125-4184, 2008.

27 M. A. Granato, J. Miguel, T. J. H. Vlugt, and A. E. Rodrigues. Diffusion Of Propane, Propylene and Isobutane In 13X Zeolite by Molecular Dynamics. Chem. Eng. Sci., 65:2656-2663, 2010.

28 C. Wang, B. Li, Y. Wang, and Z. Xie. Insight into The Topology Effect On The Diffusion Of Ethene and Propene in Zeolites: A Molecular Dynamics Simulation Study. J. Energy Chem., 22:914-918,2013.

29 G. Sastre. Computational Study of Diffusion of Propane In Small Pore Acidic Zeotypes AFX and AEI. Catal. Today, 226:25-36, 2014.

30 A. F. Combariza, D. A. Gomez, and G. Sastre. Simulating the Properties of Small Pore Silica Zeolites Using Interatomic Potentials. Chem. Soc. Rev., 42:114-127, 2013.

31 A. J. O'Malley and C. R. A. Catlow. Molecular Dynamics Simulations Of Longer N-Alkanes in Silicalite: A Comparison Of Framework And Hydrocarbon Models. Phys. Chem. Chem. Phys., 15(43):19024-19030, 2013.

32 A. J. O’Malley and C. R. A. Catlow. Molecular Dynamics Simulations Of Longer N-Alkanes In Silicalite: State-Of-The-Art Models Achieving Close Agreement With Experiment. Phys. Chem. Chem. Phys., 17(3):1943-1948, 2015.

33 R. V. Awati, P. I. Ravikovitch, and D. S. Sholl. Efficient And Accurate Methods For CharacterizingEffects Of Framework Flexibility on Molecular Diffusion In Zeolites: CH4 Diffusion In Eight Member Ring Zeolites. J. Phys. Chem. C, 117(26):13462-13473, 2013.

34 R. Krishna and J. M. van Baten. A Molecular Dynamics Investigation Of The Diffusion Character-Istics of Cavity-Type Zeolites With 8-Ring Windows. Micropor. Mesopor. Mater., 137(13):83-91, 2011.

35 A. García-Sánchez, D. Dubbeldam, and S. Calero. Modeling Adsorption And Self-Diffusion Of Methane In Lta Zeolites: The Influence Of Framework Flexibility. J. Phys. Chem. C, ACS Paragon 32 Jus Environment 
114(35):15068-15074, 2010.

36 S. Jakobtorweihen, M. G. Verbeek, C. P. Lowe, F. J. Keil, and B. Smit. Understanding The Loading Dependence Of Self-Diffusion In Carbon Nanotubes. Phys. Rev. Lett., 95:044501, 2005.

37 S. Jakobtorweihen, C. P. Lowe, F. J. Keil, and B. Smit. A Novel Algorithm To Model The Influence Of Host Lattice Flexibility In Molecular Dynamics Simulations: Loading Dependence Of Self-Diffusion In Carbon Nanotubes. J. Chem. Phys., 124:154706, 2006.

38 S. Jakobtorweihen, C. P. Lowe, F. J. Keil, and B. Smit. Diffusion Of Chain Molecules And Mixtures In Carbon Nanotubes: The Effect Of Host Lattice Flexibility And Theory Of Diffusion In The Knudsen Regime. J. Chem. Phys., 127:024904, 2007.

39 N. E. R. Zimmermann, M. Haranczyk, M. Sharma, B. Liu, B. Smit, and F. J. Keil. Adsorption And Diffusion In Zeolites: The Pitfall Of Isotypic Crystal Structures. Mol. Simul., 37:986-989, 2011.

40 B. Smit and R. Krishna. Molecular Simulations In Zeolitic Process Design. Chemical Engineering Science, 58(36):557-568, 2003.

41 S. E. Boulfelfel, P. I. Kavikovitch, and D. S. Sholl. Modeling Diffusion Of Linear Hydrocarbons In Silica Zeolite LTA Using Transition Path Sampling. J. Phys. Chem. C., 119:15643-15653, 2015.

${ }^{42}$ M. K. F. Abouelnasr and B. Smit. Diffusion In Confinement: Kinetic Simulations Of SelfAnd Collective Diffusion Behavior Of Adsorbed Gases. Phys. Chem. Chem. Phys., 14(33):1160011609, 2012.

43 P. Demontis and G. B. Suffritti. A Comment On The Flexibility Of Framework In Molecular Dynamics Simulations Of Zeolites. Micropor. Mesopor. Mater., 125(12):160-168, 2009.

44 S. Fritzsche, M. Wolfsberg, and R. Haberlandt. The Importance of Various Degrees Of Freedom In the Theoretical Study Of The Diffusion Of Methane In Silicalite-1. Chemical Physics, 289(23):321-333, 2003.

45 N. E. R. Zimmermann, S. Jakobtorweihen, E. Beerdsen, B. Smit, and F. J. Keil. In-Depth Study Of The Influence Of Hostframework Flexibility On The Diffusion Of Small Gas Molecules In One-Dimensional Zeolitic Pore Systems. J. Phys. Chem. C, 111(46):17370-17381, 2007. 
46 R. Krishna and J. M. van Baten. Comment on "Modeling Adsorption And Self-Diffusion Of Methane In Lta Zeolites: The Influence Of Framework Flexibility”. J. Phys. Chem. C, 114(41):18017-18021, 2010.

47 G. Sastre and J. D. Gale. ZeoTsites: A Code For Topological And Crystallographic Tetrahedral Sites Analysis In Zeolites And Zeotypes. Micropr. Mesopor. Mater., 43:27-40, 2001.

48 G. Sastre and A. Corma. Topological Descriptor For Oxygens In Zeolites. Analysis Of Ring Counting In Tetracoordinated Nets. J. Phys. Chem. C, 113:6398-6405, 2009.

49 J. Chen, J. M. Thomas, P. A. Wright, and R. P. Townsend. Silicoaluminophosphate Number Eighteen (SAPO-18): A New Microporous Solid Acid Catalyst. Catal. Lett., 28(2-4):241-248, 1994.

50 N. Katada, K. Nouno, J. K. Lee, J. Shin, S. B. Hong, and M. Niwa. Acidic Properties Of Cage- Based, Small-Pore Zeolites With Different Framework Topologies And Their Silicoaluminophosphate Analogues. J. Phys. Chem. C, 115(45):22505-22513, 2011.

51 S. T. Wilson, R. W. Broach, C. S. Blackwell, C. A. Bateman, N. K. McGuire, and R. M. Kirchner. Synthesis, Characterization And Structure Of SAPO-56, A Member Of The Abc Double- Six-Ring Family Of Materials With Stacking Sequence aabbccbb. Micropor. Mesopor. Mater., 28(1):125-137, 1999.

52 G. Sastre, D. W. Lewis, and C. R. A. Catlow. Structure And Stability Of Silica Species In SAPO

Molecular Sieves. J. Phys. Chem., 100(16):6722-6730, 1996.

53 G. Sastre, D. W. Lewis, and C. R. A. Catlow. Modeling Of Silicon Substitution In SAPO-5 And SAPO-34 Molecular Sieves. J. Phys. Chem. B, 101(27):5249-5262, 1997.

54 K. Hemelsoet, A. Ghysels, D. Mores, K. De Wispelaere, V. Van Speybroeck, B. M. Weckhuysen, and M. Waroquier. Experimental And Theoretical IR Study Of Methanol And Ethanol Conversion Over H-SAPO-34. Cat. Today, 177(1):12-24, 2011.

55 P. Demontis and G. B. Suffritti. Structure And Dynamics Of Zeolites Investigated By Molecular Dynamics. Chem. Rev., 97(8):2845-2878, 1997.

56 R. Krishna. Diffusion In Porous Crystalline Materials. Chem. Soc. Rev., 41:3099-3118, 2012.

57 W. Smith, C. W. Yong, and P. M. Rodger. DL_POLY: Application To Molecular Simulation. 
Mol. Simul., 28(5):385-471, 2002.

Y. G. Bushuev and G. Sastre. Atomistic Simulations Of Water And Organic Templates Occluded During The Synthesis Of Zeolites. Micropor. Mesopor. Mater., 129(12):42-53, 2010.

59 Y. G. Bushuev and G. Sastre. Atomistic Simulations Of Structural Defects And Water Occluded in ssz-74 Zeolite. J. Phys. Chem. C, 113(25):10877-10886, 2009.

60 J. D. Gale and N. J. Henson. Derivation Of Interatomic Potentials For Microporous Aluminophos- Phates From The Structure And Properties Of Berlinite. J. Chem. Soc., Faraday Trans., 90:3175-3179, 1994.

61 K.-P. Schröder, J. Sauer, M. Leslie, C. Richard, A. Catlow, and J. M. Thomas. Bridging Hydroxyl Groups In Zeolitic Catalysts: A Computer Simulation Of Their Structure, Vibrational Properties And Acidity In Protonated Faujasites (H-Y Zeolites). Chem. Phys. Lett., 188(34):320-325, 1992.

62 J. D. Gale and A. L. Rohl. The General Utility Lattice Program (Gulp). Mol. Simul., 29(5):291-341, 2003.

63 T. Oie, G. M. Maggiora, R. E. Christoffersen, and D. J. Duchamp. Development Of A Flexi- Ble Intra- And Intermolecular Empirical Potential Function For Large Molecular Systems. Int. J. Quantum Chem., 20(S8):1-47, 1981.

64 A. F. Combariza, G. Sastre, and A. Corma. Molecular Dynamics Simulations Of The Diffusion Of Small Chain Hydrocarbons In 8-Ring Zeolites. J. Phys. Chem. C, 115(4):875-884, 2011.

65 W. Smith and T.R. Forester._ DL POLY 2.0: A General-Purpose Parallel Molecular Dynamics Simulation Package. J. Mol. Graph., 14(3):136-141, 1996.

66 P. Tschaufeser and S. C. Parker. Thermal Expansion Behavior Of Zeolites And Alpo4s. J. Phys Chem., 99(26):10609-10615, 1995.

67 D. A. Woodcock, P. Lightfoot, L. A. Villaescusa, M.-J. Díaz-Cabañas, M. A. Camblor, And D. Engberg. Negative Thermal Expansion In The Siliceous Zeolites Chabazite And Itq-4: A Neutron Powder Diffraction Study. Chem. Mater., 11(9):2508-2514, 1999.

68 J. D. Gale. Analytical Free Energy Minimization Of Silica Polymorphs. J. Phys. Chem. B, 102(28):5423-5431, 1998.

69 M. M. Martínez-Iñesta and R. F. Lobo. Investigation Of The Negative Thermal Expansion Mech- Anism Of Zeolite Chabazite Using The Pair Distribution Function Method. J. Phys. 
Chem. B, 109(19):9389-9396, 2005.

70 B. W. H. van Beest, G. J. Kramer, and R. A. van Santen. Force Fields For Silicas And Alu- Minophosphates Based On Ab Initio Calculations. Phys. Rev. Lett., 64(16):1955-1958, 1990.

71 A. F. Combariza, G. Sastre, and A. Corma. Propane/Propylene Diffusion In Zeolites: Framework Dynamics. J. Phys. Chem. C, 113:11246-11253, 2009.

72 N. Hedin, G. J. DeMartin, W. J. Roth, K. G. Strohmaier, and S. R. Reyes. Experimental SelfDiffusion Coefficients Of Methane, Ethene, Ethane And Propene in DDR, CHA and LTA. Micropor. Mesopor. Mater., 109:327, 2008.

73 D. H. Olson, M. A. Camblor, L. A. Villaescusa, and G. H. Kuehl. Light Hydrocarbon Sorption Properties Of Pure Silica Si-Cha And Itq-3 And High Silica zsm-58. Micropor. Mesopor. Mat., 67:27-33, 2004.

74 D. M. Ruthven and S. C. Reyes. Adsorptive Separation Of Light Olefins From Paraffins. Micropor. Mesopor. Mat., 104:59-66, 2007.

75 N. A. Ramsahye and R. G. Bell. Calculating The Energy Barriers To Sodium Cation Motion Through The Six-Rings Of Zeolite Y. Micropor. Mesopor. Mater., 109:405-412, 2007.

76 J. Chen and C. L. Brooks III. Implicit Modeling Of Nonpolar Solvation For Simulating Protein Folding And Conformational Transitions. Phys. Chem. Chem. Phys., 10:471-481, 2008. 